\title{
DISTRIBUTION OF CHEMICAL ELEMENTS IN SOIL FROM CRN DRIM RIVER BASIN, REPUBLIC OF MACEDONIA ${ }^{\#}$
}

\author{
Trajče Stafilov ${ }^{*}$, Robert Šajn $^{2}$, Ivana Mickovska ${ }^{1}$ \\ ${ }^{1}$ Institute of Chemistry, Faculty of Natural Sciences and Mathematics, Ss Cyril and Methodius University, \\ Skopje, Republic of Macedonia \\ ${ }^{2}$ Geological Survey of Slovenia, Ljubljana, Slovenia \\ e-mail: trajcest@pmf.ukim.mk
}

\begin{abstract}
The aim of this study was to investigate the distribution of chemical elements in topsoil and subsoil, focusing on the identification of natural and anthropogenic element sources in the area of the Crn Drim River Basin, Republic of Macedonia. For that purpose, by using sampling network of $5 \times 5 \mathrm{~km}, 124$ soil samples from 62 locations (topsoil and bottom soil) were collected. In total 60 elements were analysed, from which 18 elements (Ag, $\mathrm{Al}, \mathrm{B}, \mathrm{Ba}, \mathrm{Ca}, \mathrm{Cr}, \mathrm{Cu}, \mathrm{Fe}$, $\mathrm{K}, \mathrm{Li}, \mathrm{Mg}, \mathrm{Mn}, \mathrm{Na}, \mathrm{Ni}, \mathrm{P}, \mathrm{Pb}, \mathrm{V}$ и $\mathrm{Zn}$ ) were analysed by inductively coupled plasma - atomic emission spectrometry (ICP-AES) and an additional 42 elements were analysed by ICP - mass spectrometry (ICP-MS). Multivariate statistical analysis was applied to the obtained data. Factor analysis applied to the ICP-AES results produced four geogenic factors: F1 (Ba and K); F2 (Ag, Cd, Cu, Ni, Pb and Zn), F3 (Cr, Fe, Na, Ni and V) and F4 (Al, Ca, Mg and Mn). Data obtained from the distribution maps and data analysis on soil samples, indicate the natural occurrence of the analysed elements as well as low concentrations of heavy metals in the studied area.
\end{abstract}

Key words: soil; Crn Drim River Basin; Republic of Macedonia; multivariate statistics; geochemical mapping

\section{INTRODUCTION}

Pollution with heavy metals is a global problem initiated by progress in world technology and the human exploitation of natural resources; this has become a subject of many studies. The regional contamination of soil occurs mainly in industrial regions and within large settlement centres, where factories, traffic and municipal waste are the most important sources of trace metals [1]. The level of environmental pollution depends on the proper control of anthropogenic activities; these factors indicate a global problem of environmental pollution [2].

Heavy metals occur as natural constituents of the Earth's crust, and are persistent environmental contaminants since they cannot be degraded or destroyed. Some heavy metals have bio-importance as trace elements, but the biotoxic effects of many on human biochemistry are of great concern. Hence, there is a need for the proper understanding of the conditions, such as concentrations and oxidation states, which make them harmful, and how biotoxicity occurs [3]. The major causes of emission are anthropogenic sources, specifically mining operations, where, in some cases, even long after mining activities have ceased, the emitted metals continue to persist in the environment [4-6].

The soil cover of the Republic of Macedonia is very heterogeneous, with great changes over small distances. Almost all of the relief forms, geological formations, climatic influences, plant associations and soils that appear in Europe (with the exception of podzols) are represented. More than 30 soil types are found in Macedonia [7-9]. According to Filipovski [10] negative degradation of soils in Macedonia started a long time ago. In recent years, results suggest that the Republic of Macedonia has the same problem of pollution by heavy metals, and the most important emission sources are mines, 
drainage systems and smelters near the towns of Veles, Tetovo, Kavadarci and Probištip [11-21].

The goal of the present study was to investigate the spatial distribution of about 60 chemical elements in soil from the Crn Drim River Basin, in the Republic of Macedonia. For this purpose, applying a sampling network of $5 \times 5 \mathrm{~km}, 124$ soil samples from 62 locations (topsoil and bottom soil) were collected. The soil samples were analysed by inductively coupled plasma - atomic emission spectrometry (ICP-AES) for macro-elements $(\mathrm{Ag}, \mathrm{Al}, \mathrm{B}$, $\mathrm{Ba}, \mathrm{Ca}, \mathrm{Cr}, \mathrm{Cu}, \mathrm{Fe}, \mathrm{K}, \mathrm{Li}, \mathrm{Mg}, \mathrm{Mn}, \mathrm{Na}, \mathrm{Ni}, \mathrm{P}, \mathrm{Pb}, \mathrm{V}$ и $\mathrm{Zn)}$ and inductively coupled plasma - mass spectrometry (ICP-MS) for trace elements (As, Be, Bi, $\mathrm{Br}, \mathrm{Cd}, \mathrm{Ce}, \mathrm{Co}, \mathrm{Cs}, \mathrm{Dy}, \mathrm{Er}, \mathrm{Eu}, \mathrm{Ga}, \mathrm{Gd}, \mathrm{Ge}, \mathrm{Hf}, \mathrm{Ho}$, I, In, La, Lu, Mo, Nb, Nd, Pd, Pr, Pt, Rb, Sb, Sc, $\mathrm{Sm}, \mathrm{Sn}, \mathrm{Sr}, \mathrm{Ta}, \mathrm{Tb}, \mathrm{Te}, \mathrm{Ti}, \mathrm{Tl}, \mathrm{Tm}, \mathrm{W}, \mathrm{Y}, \mathrm{Yb}$ и Zr).

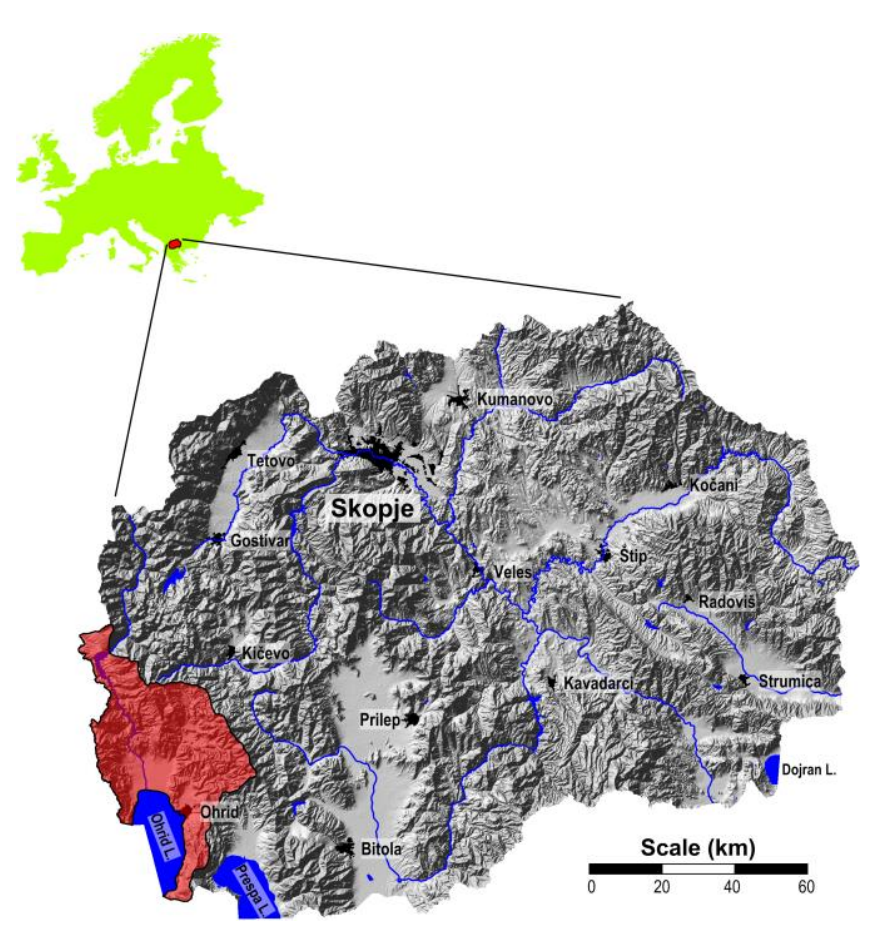

Figure 1. The investigated area on the map of the Republic of Macedonia

In the far southwest of the Republic of Macedonia, the Ohrid-Struga valley stretches between the Mts. of Jablanica, Belička and Mokra in the west; Mts. Galičica, Petrina, Plaćenska and Ilinska in the east; Stogovo and its branch Karaorman to the north and the hilly region of Gora to the south. It is characterised by a changed Mediterranean climate and winds coming from the west and north. The last valley is the Debarca valley, located to the north of the Ohrid valley [24].

\section{STUDY AREA}

The investigated area is located in the southwestern part of Macedonia and covers the basin of the Crn Drim River (Figures 1 and 2). This river passes through two valleys: Ohrid-Struga and the Debar-Radika River basin. Crn Drim flows from the Ohrid Lake in the town of Struga. First it flows through Struga Field to the village of Tašmarunište, where it enters into the Drimkolska Gorge and immediately in the artificial Globočica Lake. After the Globočica dam, a short river flow is again formed to re-enter the nearby Debar Lake. It leaves it at Debar Lake dam and continues $12 \mathrm{~km}$ as a border river and then enters into Albania. The Crn Drim River Basin covers an area of $3,350 \mathrm{~km}^{2}$. The area receives an average of $933 \mathrm{~mm}$ of precipitation per year [22, 23].

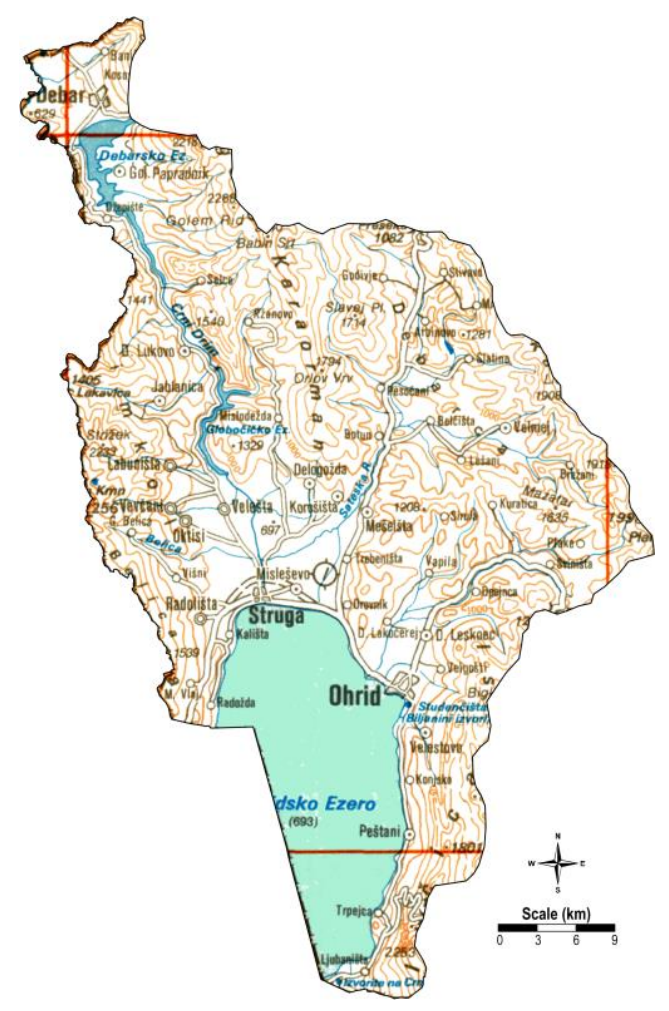

Figure 2. Topographic map of the investigated area

The largest part of the Crn Drim River Basin is tectonically separated as part of the WestMacedonian zone with properties formed during the Hercynian and Alpine orogeny. During the Hercynian orogeny, Paleozoic metamorphism was formed in synclines and anticlines, while the Alpine orogeny was characterised by intense metamorphism. Later in this orogeny, Tertiary tectonic trenches were formed. Triassic sediments were formed during the Alpine orogeny, in huge structures orientated mostly 
in the north-south and northwest-southeast directions [25-28].

The area of Debar and low flow of the Radika River (Debar zone) is in the Cukali-Krasta zone [25-28]. The Cukali-Krasta zone is composed of Upper Cretaceous (i.e. Turonian) conglomerates: sandstones, claystones and limestones with olistostromes. In this zone, evaporites and minor Paleogene sediments are present. The boundary with the West-Macedonian zone is marked by the Stogovo nappe, after which the Paleozoic, Triassic and Jurassic complex occurs as a bundled mass; it has an allochthonous length of at least $7-10 \mathrm{~km}$ through the Upper Cretaceous sediments and evaporites of the Cukali-Krasta zone. This zone of the Debar area essentially represents a tectonic half-window. The diapiric structure of Dešat Mountain is very important in the Cukali-Krasta zone; it is composed of anhydrite and gypsum, characterised by partial but noticeable diapiric, internal folding and brecciation, with the development of tectonic breccias - mylonites at the contacts with the adjacent rock masses.

The geological structure of the investigated area is dominated by alluvial creations and semisedimentary sediments, distributed in the plains (Figure 3). This area is located in the WestMacedonian geotectonic zone, within the Ohrid Neogene Basin. In the bases of the Neogene and Quaternary sediments lies the Triassic rocks, while on the surface of the terrain are the lake and swamp sediments that are deposited in the Quaternary period; here they have the greatest distribution [25-28].

The creation of this graben structure is related to the end of the lower and early middle Pliocene: that is, the period when the expansion of the orogenic phase began with the manifestation of intense differentiated vertical movements. As a result of such processes, in the beginning, the old fault structures were reactivated and later on, they came to be a sink in the space (i. e. its transformation into Pliocene lake basins) [25, 29].

The Pliocene sediments in this area are found in the southern part, as well as in the northwest part. The Galičica Mountain is a dominant relief appearance located between Ohrid Lake to the west and Prespa Lake to the east. According to its structural characteristics, Galičica Mountain is a typical horst that has been elevated by the dominant neotectonic interblock faults.

The maximum vertical movement in the western part of Mt. Galičica was accomplished via the system of scalable faults. Due to the massiveness of limestones and the intensive radial tectonics, with which the mountain is cut into several blocks, the dials cannot be noticed. In the lower parts, the synclinal structure is constructed of Triassic conglomerates that lie over the Paleozoic metamorphites [25-28].

The area of Ohrid is characterised by Paleozoic, Triassic, Jurassic, Neocene and Quaternary formations. The Paleozoic formations consist of a thick complex of metamorphic and magmatic rocks. Based on fossil remains, this complex is divided over the Cambrian, Ordovician and Devonian periods. The Cambrian and Ordovian sections are composed of phyllite schists, and the Devonian is characterised by phylitic shales, metaconglomerates, carbon shales and marble limestones. Through the Paleozoic metamorphism, granodiorites and syenites were intertwined. The Triassic formations lie transgressive over the Paleozoic complex. In some localities (Mt. Jablanica), the Triassic sediments are overlapped with conglomerates composed of diabase, gabbro, Triassic limestone and, in the final boundaries, serpentinites and shales. Neocene sediments have the character of fresh water, and with their microflora are assumed to have formed during the Middle Pliocene period. There are also sediments of the new Pliocene. Quaternary sediments are widespread and consist of glacial, morene, and fluvioglacial sediments, Terra Rosa, travertine, breccias, thallus and alluvial deposits [29].

\section{EXPERIMENTAL}

\section{Sampling}

Samples of natural surface soils in the Crn Drim River Basin, in the Republic of Macedonia, were collected according to the European guidelines for soil pollution studies [30], and also according to our experience $[12,13,20,31]$. The study area $\left(3350 \mathrm{~km}^{2}\right)$ was covered by a sampling grid of $5 \times 5$ $\mathrm{km}$ (Figure 4). Altogether 124 soil samples were collected from 62 locations, with the collection of topsoil $(0-5 \mathrm{~cm})$ and subsoil $(20-30 \mathrm{~cm})$ samples. The possible organic horizon was excluded. One sample represents the composite material collected at the central sample point itself, and at least four points within a radius of $10 \mathrm{~m}$ around it towards the north, east, south and west. The mass of the composite sample was about $1 \mathrm{~kg}$. 


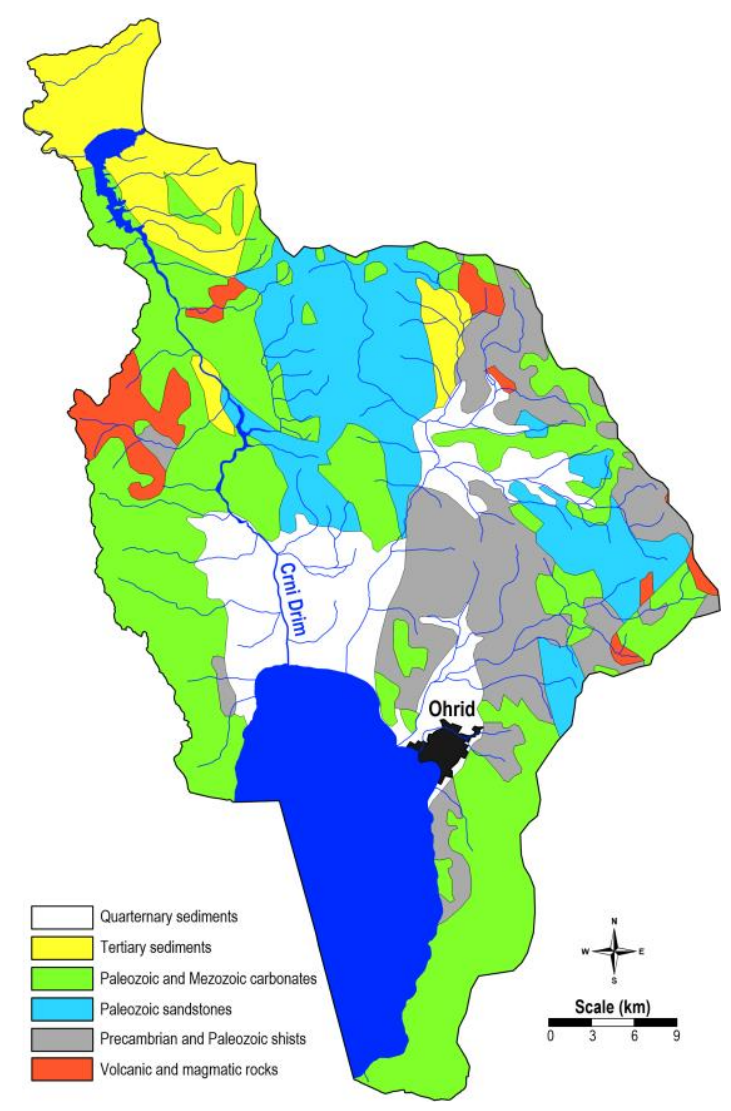

Figure 3. General geological map of the Crn Drim River Basin

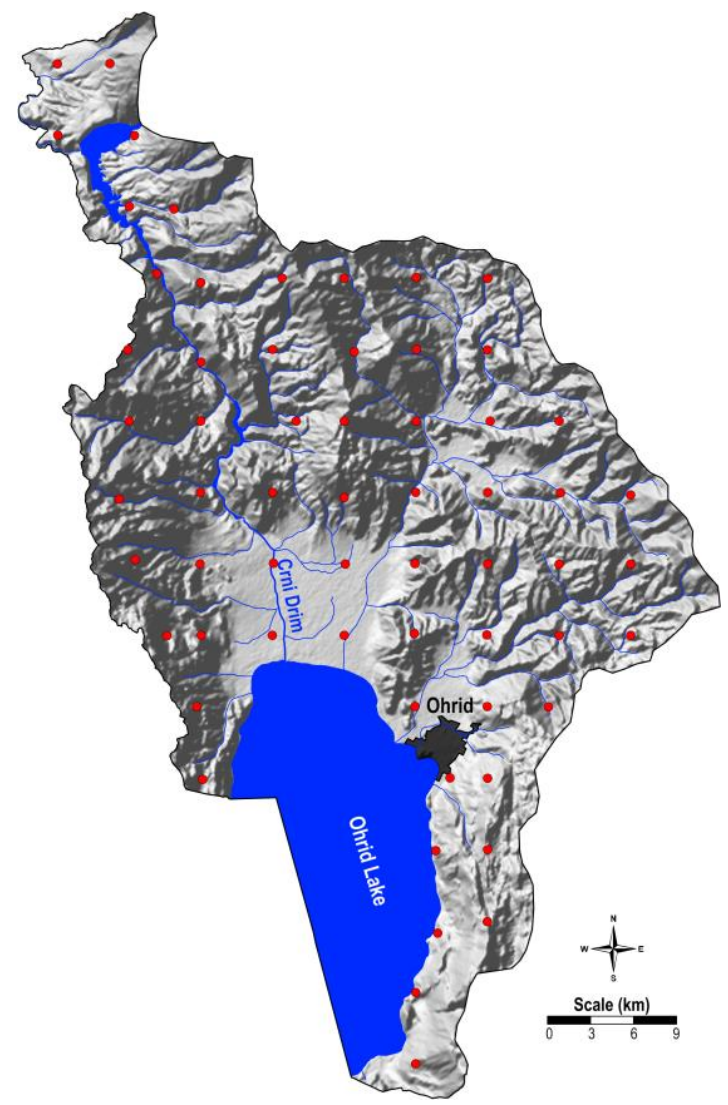

Figure 4. Soil sampling locations in the Crn Drim River Basin Sample preparation 
The soil samples were air dried indoors at room temperature for about two weeks. They were then gently crushed, cleaned of extraneous material and sifted through a plastic sieve with $2 \mathrm{~mm}$ mesh [30]. The sifted mass was quartered and milled in an agate mill to produce an analytical grain size below $0.125 \mathrm{~mm}$.

For the digestion of soil samples, open wet digestion with a mixture of acids was applied. The digestion was carried out in this order: the precisely measured mass of dust samples $(0.25 \mathrm{~g})$ was placed in Teflon vessels. After this, $5 \mathrm{ml}$ of concentrated $\mathrm{HNO}_{3}$ was added, until brown vapours left the vessels. Nitric acid is a very suitable oxidant for the digestion of organic matter in samples. For the total digestion of inorganic components, 5-10 ml HF was added. When the digest became a clear solution, 2 $\mathrm{ml}$ of $\mathrm{HClO}_{4}$ was added for the total digestion of organic matter. After cooling the vessels for $15 \mathrm{~min}$, $2 \mathrm{ml}$ of $\mathrm{HCl}$ and $5 \mathrm{ml}$ of water were added for the total dissolution of metal ions. Finally, the vessels were cooled and digests were quantitatively transferred to $50 \mathrm{ml}$ calibrated flasks [20].

\section{Instrumentation}

All samples were analysed by ICP-AES (Varian, model 715-ES) for the elements with higher contents according to the instrumental conditions presented by Balabanova et al. [14]. Trace elements were analysed by ICP-MS measurements on a SCIEX Perkin Elmer Elan DRC II (Canada) inductively coupled plasma mass spectrometer (with quadruple and single detector setup) under the instrumental conditions presented earlier [16]. The quality control of the applied techniques was performed using the standard addition method, and the recovery for the investigated elements ranged from $98.2 \%$ to $100.8 \%$.

\section{Data processing}

Data analysis and the production of maps were performed on a PC using Paradox (ver. 9), Statistica (ver. 6.1), AutoDesk Fig. (ver. 2008) and Surfer (ver. 8.09) software. All field observations, analytical data and measurements were introduced into the data matrix. Parametric and nonparametric statistical methods were used for data analysis [20]. Based on the results of the normality tests and visual inspection of the distribution histograms, for all elements, logarithms of the element content were used to acquire normal distributions. Basic statistics for all 60 elements in the topsoil and bottom soil are given in Tables 1 and 2.
Multivariate R-mode factor analysis [20] was used to reveal associations of the chemical elements that had been determined by ICP-AES. From numerous variables, the factor analysis (FA) derives a smaller number of new, synthetic variables. Factor analysis was performed on variables standardised to a mean of zero and one unit of standard deviation [32]. As a measure of similarity between variables, the product-moment correlation coefficient (r) was applied. For orthogonal rotation, the varimax method was used.

The universal kriging method with linear variogram interpolation was applied for the construction of the areal distribution maps of the 18 elements determined by ICP-AES and the factor scores (F1-F4) for topsoil $(0-5 \mathrm{~cm})$ and subsoil $(20-30 \mathrm{~cm})$ samples. Seven classes of the following percentile values were selected: $0-10,10-25,25-$ $40,40-60,60-75,75-90$ and 90-100.

\section{RESULTS AND DISCUSSION}

ICP-AES was applied for the analysis of 18 macro-elements (Ag, Al, B, Ba, Ca, Cr, $\mathrm{Cu}, \mathrm{Fe}, \mathrm{K}, \mathrm{Li}$, $\mathrm{Mg}, \mathrm{Mn}, \mathrm{Na}, \mathrm{Ni}, \mathrm{P}, \mathrm{Pb}, \mathrm{V}$ и $\mathrm{Zn}$ ) while the additional 48 elements (As, Be, Bi, Br, Cd, Ce, Co, Cs, Dy, Er, $\mathrm{Eu}, \mathrm{Ga}, \mathrm{Gd}, \mathrm{Ge}, \mathrm{Hf}, \mathrm{Ho}$, I, In, La, Lu, Mo, Nb, Nd, Pd, Pr, Pt, Rb, Sb, Sc, Sm, Sn, Sr, Ta, Tb, Te, Ti, Tl, Tm, $\mathrm{W}, \mathrm{Y}, \mathrm{Yb}$ и $\mathrm{Zr}$ ) were analysed by inductively coupled plasma - mass spectrometry (ICP-MS).

Descriptive statistics of the measurements for topsoil and bottom soil samples from 62 locations (124 soil samples total) are presented in Tables 1 and 2. Values for $\mathrm{Al}, \mathrm{Ca}, \mathrm{Fe}, \mathrm{K}, \mathrm{Mg}, \mathrm{Na}$ and $\mathrm{Ti}$ are in $\%$, values for I, Lu, In, Te and $\mathrm{Tm}$ are in $\mu \mathrm{g} / \mathrm{kg}$, and the values for the remaining elements are in $\mathrm{mg} / \mathrm{kg}$. In Tables 1 and 2 , the following statistical parameters are given: $\mathrm{X}$ - arithmetic average; $\mathrm{X}(\mathrm{BC})$ - arithmetic average after Box-Cox method; $\mathrm{Md}$ - median; min - minimum; max - maximum; $\mathrm{P}_{10}-10$ th percentile; $\mathrm{P}_{90}-90$ th percentile; $\mathrm{P}_{25}-25$ th percentile; $\mathrm{P}_{75}-75$ th percentile; $\mathrm{S}$ - standard deviation; $\mathrm{Sx}$ - standard error; $\mathrm{CV}$ - coefficient of variation; A - skewness; E - kurtosis; BC - Box-Cox transformed values.

The order of the distribution of concentrations of the major elements $\mathrm{Al}, \mathrm{Ca}, \mathrm{Fe}, \mathrm{K}, \mathrm{Mg}, \mathrm{Na}$ and $\mathrm{Ti}$ were in the following ranges: $0.42-6.5 \% \mathrm{Al} ; 0.09$ $4.7 \% \mathrm{Ca} ; 2.2-6.7 \% \mathrm{Fe} ; 0.16-2.4 \% \mathrm{~K} ; 0.21-7.2 \%$ $\mathrm{Mg}$, and $0.027-0.68 \% \mathrm{Na}$ and $0.10-0.84 \% \mathrm{Ti}$. The contents of major elements are most frequently a result of the dominant geological formations of the area: Quaternary sediments, Precambrian and Paleozoic schists, Paleozoic sandstones and Paleozoic and Mesozoic carbonates. 
Table 1. Descriprive statistics for the content of analyzed elements in top-soil samples the Crn Drim River Basin

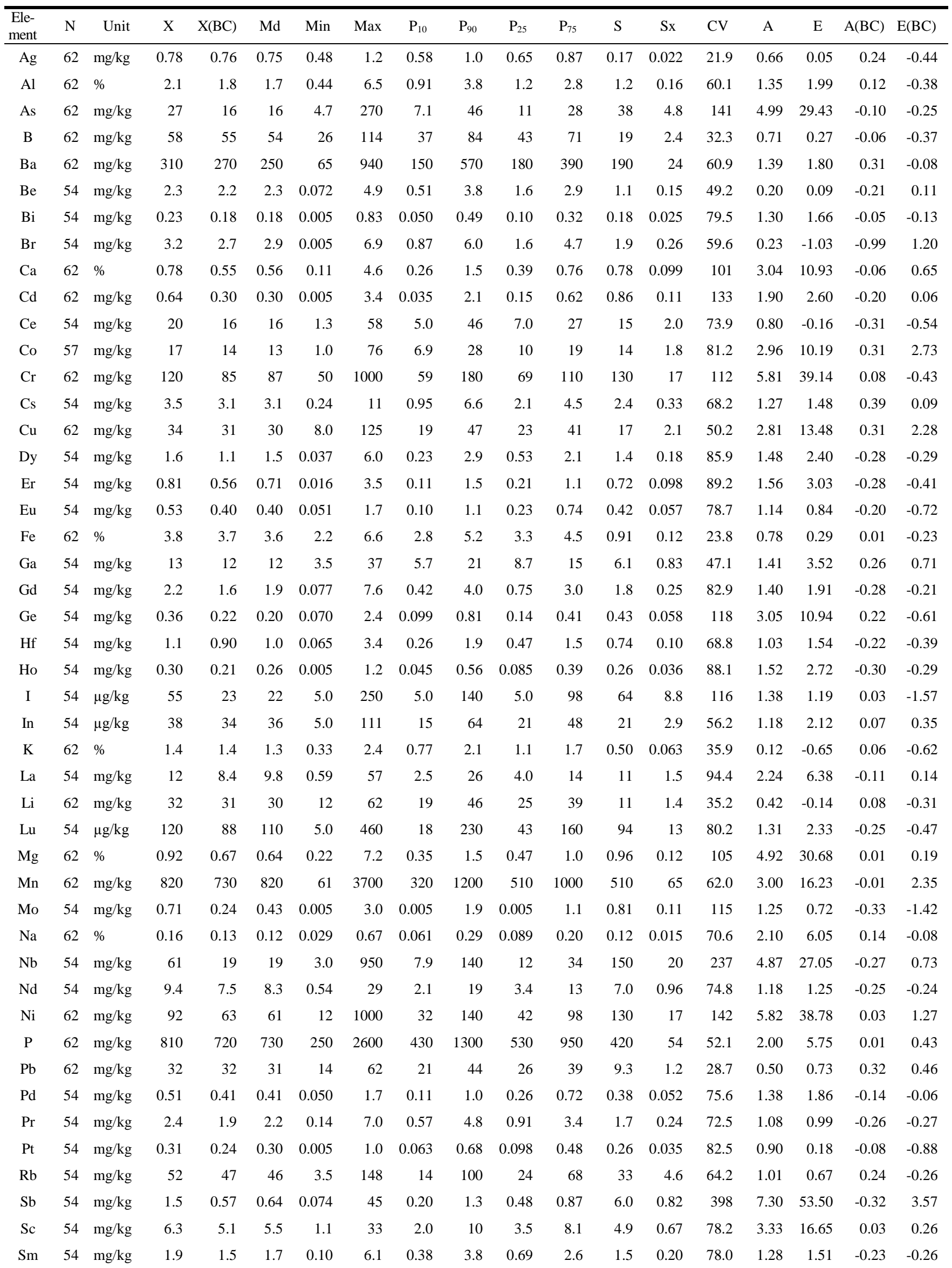


Table 1 (continuation)

\begin{tabular}{|c|c|c|c|c|c|c|c|c|c|c|c|c|c|c|c|c|c|c|}
\hline Sn & 54 & $\mathrm{mg} / \mathrm{kg}$ & 17 & 11 & 10 & 0.30 & 141 & 2.2 & 36 & 5.8 & 24 & 21 & 2.9 & 124 & 3.89 & 20.61 & -0.19 & 0.60 \\
\hline $\mathrm{Sr}$ & 54 & $\mathrm{mg} / \mathrm{kg}$ & 28 & 25 & 27 & 0.88 & 79 & 9.6 & 46 & 17 & 39 & 17 & 2.3 & 58.8 & 0.88 & 0.97 & -0.47 & 0.70 \\
\hline $\mathrm{Ta}$ & 54 & $\mathrm{mg} / \mathrm{kg}$ & 1.0 & 0.62 & 0.75 & 0.050 & 7.6 & 0.16 & 2.0 & 0.36 & 1.2 & 1.2 & 0.16 & 117 & 3.59 & 16.66 & -0.80 & 1.08 \\
\hline $\mathrm{Tb}$ & 54 & $\mathrm{mg} / \mathrm{kg}$ & 0.29 & 0.22 & 0.26 & 0.005 & 1.0 & 0.051 & 0.53 & 0.10 & 0.41 & 0.25 & 0.034 & 83.9 & 1.43 & 2.13 & -0.32 & -0.05 \\
\hline $\mathrm{Te}$ & 54 & $\mu \mathrm{g} / \mathrm{kg}$ & 27 & 11 & 5.0 & 5.0 & 200 & 5.0 & 64 & 5.0 & 40 & 38 & 5.2 & 144 & 2.92 & 10.00 & 0.40 & -1.54 \\
\hline $\mathrm{Ti}$ & 54 & $\%$ & 0.32 & 0.29 & 0.29 & 0.10 & 0.84 & 0.18 & 0.49 & 0.23 & 0.36 & 0.14 & 0.019 & 44.2 & 1.45 & 3.18 & 0.16 & 0.46 \\
\hline $\mathrm{Tl}$ & 54 & $\mathrm{mg} / \mathrm{kg}$ & 0.46 & 0.36 & 0.38 & 0.050 & 1.5 & 0.050 & 0.90 & 0.17 & 0.71 & 0.35 & 0.047 & 76.4 & 0.84 & 0.22 & -0.18 & -0.91 \\
\hline $\mathrm{Tm}$ & 54 & $\mu \mathrm{g} / \mathrm{kg}$ & 110 & 82 & 100 & 5.0 & 480 & 16 & 220 & 33 & 150 & 100 & 14 & 87.5 & 1.50 & 2.82 & -0.18 & -0.63 \\
\hline V & 62 & $\mathrm{mg} / \mathrm{kg}$ & 110 & 99 & 98 & 65 & 410 & 71 & 150 & 87 & 120 & 48 & 6.2 & 44.0 & 4.21 & 24.67 & 0.00 & -0.09 \\
\hline W & 54 & $\mathrm{mg} / \mathrm{kg}$ & 1.4 & 1.1 & 1.1 & 0.064 & 4.8 & 0.20 & 2.8 & 0.61 & 2.1 & 1.1 & 0.14 & 74.1 & 0.97 & 0.88 & -0.19 & -0.55 \\
\hline Y & 54 & $\mathrm{mg} / \mathrm{kg}$ & 7.2 & 5.3 & 4.9 & 0.22 & 29 & 1.6 & 12 & 2.8 & 11 & 6.1 & 0.83 & 84.8 & 1.63 & 3.01 & -0.24 & 0.06 \\
\hline $\mathrm{Yb}$ & 54 & $\mathrm{mg} / \mathrm{kg}$ & 0.73 & 0.52 & 0.66 & 0.018 & 3.1 & 0.11 & 1.4 & 0.22 & 0.99 & 0.63 & 0.085 & 86.4 & 1.48 & 2.82 & -0.25 & -0.49 \\
\hline $\mathrm{Zn}$ & 62 & $\mathrm{mg} / \mathrm{kg}$ & 140 & 130 & 130 & 58 & 290 & 90 & 210 & 110 & 170 & 52 & 6.5 & 36.1 & 1.05 & 1.03 & -0.20 & 0.34 \\
\hline $\mathrm{Zr}$ & 54 & $\mathrm{mg} / \mathrm{kg}$ & 58 & 53 & 56 & 7.1 & 144 & 21 & 95 & 37 & 78 & 30 & 4.1 & 51.7 & 0.66 & 0.42 & -0.25 & -0.17 \\
\hline
\end{tabular}

$\mathrm{N}$ - Number of samples; $\mathrm{X}$ - mean; $\mathrm{X}(\mathrm{BC})$ - mean of Box-Cox transformed values; Md - median; Min - minimum;

Max - maximum; $\mathrm{P}_{10}-10^{\text {th }}$ percentile; $\mathrm{P}_{25}-25^{\text {th }}$ percentile; $\mathrm{P}_{75}-75^{\text {th }}$ percentile $; \mathrm{P}_{90}-90^{\text {th }}$ percentile; $\mathrm{S}-$ standard deviation; $\mathrm{S}_{\mathrm{x}}$ - standard deviation of transformed values; CV - coefficient of variation, A - skewness; E - kurtosis; $\mathrm{BC}-\mathrm{Box}-\mathrm{Cox}$ transformed values

Table 2. Descriprive statistics for the content of analyzed elements in sub-soil samples from the Crn Drim River Basin

\begin{tabular}{|c|c|c|c|c|c|c|c|c|c|c|c|c|c|c|c|c|c|c|}
\hline $\begin{array}{l}\text { Ele- } \\
\text { ment }\end{array}$ & $\mathrm{n}$ & Unit & $\mathrm{X}$ & $\begin{array}{c}\mathrm{X}(\mathrm{B} \\
\mathrm{C})\end{array}$ & $\mathrm{Md}$ & Min & Max & $\mathrm{P}_{10}$ & $\mathrm{P}_{90}$ & $\mathrm{P}_{25}$ & $\mathrm{P}_{75}$ & $S$ & Sx & $\mathrm{CV}$ & A & E & $\begin{array}{c}\mathrm{A}(\mathrm{B} \\
\mathrm{C})\end{array}$ & $\begin{array}{c}\mathrm{E}(\mathrm{B} \\
\mathrm{C})\end{array}$ \\
\hline $\mathrm{Ag}$ & 62 & $\mathrm{mg} / \mathrm{kg}$ & 0.82 & 0.80 & 0.80 & 0.44 & 1.2 & 0.59 & 1.1 & 0.68 & 0.93 & 0.18 & 0.022 & 21.5 & 0.20 & -0.51 & -0.24 & -0.29 \\
\hline $\mathrm{Al}$ & 62 & $\%$ & 2.4 & 2.0 & 1.9 & 0.42 & 5.9 & 0.83 & 4.4 & 1.4 & 3.2 & 1.4 & 0.17 & 58.0 & 0.89 & 0.17 & -0.15 & -0.58 \\
\hline As & 62 & $\mathrm{mg} / \mathrm{kg}$ & 27 & 16 & 16 & 5.5 & 280 & 8.2 & 50 & 11 & 31 & 39 & 5.0 & 143 & 5.00 & 30.39 & 0.20 & -0.39 \\
\hline B & 62 & $\mathrm{mg} / \mathrm{kg}$ & 60 & 56 & 59 & 25 & 140 & 38 & 87 & 45 & 67 & 21 & 2.7 & 35.5 & 1.43 & 3.51 & 0.04 & 0.60 \\
\hline $\mathrm{Ba}$ & 62 & $\mathrm{mg} / \mathrm{kg}$ & 310 & 260 & 240 & 28 & 980 & 90 & 580 & 150 & 390 & 210 & 26 & 67.2 & 1.19 & 1.27 & -0.13 & -0.11 \\
\hline $\mathrm{Be}$ & 58 & $\mathrm{mg} / \mathrm{kg}$ & 2.3 & 2.3 & 2.1 & 0.005 & 5.3 & 0.84 & 3.9 & 1.8 & 3.1 & 1.2 & 0.16 & 50.7 & 0.36 & 0.20 & -0.08 & 0.20 \\
\hline $\mathrm{Bi}$ & 58 & $\mathrm{mg} / \mathrm{kg}$ & 0.20 & 0.16 & 0.17 & 0.005 & 0.78 & 0.048 & 0.40 & 0.10 & 0.24 & 0.15 & 0.020 & 77.9 & 1.58 & 3.03 & 0.00 & 0.42 \\
\hline $\mathrm{Br}$ & 58 & $\mathrm{mg} / \mathrm{kg}$ & 3.9 & 3.0 & 2.7 & 0.005 & 20 & 0.76 & 8.1 & 1.8 & 4.5 & 4.2 & 0.55 & 106 & 2.52 & 6.87 & 0.54 & 1.38 \\
\hline $\mathrm{Ca}$ & 62 & $\%$ & 1.0 & 0.56 & 0.54 & 0.095 & 4.7 & 0.21 & 2.7 & 0.31 & 1.0 & 1.2 & 0.15 & 117 & 1.98 & 3.10 & 0.02 & -0.28 \\
\hline $\mathrm{Cd}$ & 62 & $\mathrm{mg} / \mathrm{kg}$ & 0.83 & 0.34 & 0.29 & 0.005 & 7.3 & 0.052 & 2.1 & 0.13 & 0.94 & 1.3 & 0.17 & 160 & 2.92 & 9.90 & 0.14 & -0.07 \\
\hline $\mathrm{Ce}$ & 58 & $\mathrm{mg} / \mathrm{kg}$ & 21 & 15 & 14 & 0.37 & 105 & 2.5 & 55 & 7.9 & 29 & 22 & 2.8 & 102 & 1.97 & 4.32 & 0.15 & -0.05 \\
\hline Co & 59 & $\mathrm{mg} / \mathrm{kg}$ & 16 & 14 & 14 & 1.9 & 56 & 7.0 & 29 & 9.9 & 22 & 9.5 & 1.2 & 58.5 & 1.51 & 3.93 & -0.26 & 0.74 \\
\hline $\mathrm{Cr}$ & 62 & $\mathrm{mg} / \mathrm{kg}$ & 120 & 86 & 86 & 49 & 910 & 59 & 180 & 69 & 110 & 120 & 15 & 102 & 5.03 & 30.75 & 0.23 & -0.43 \\
\hline Cs & 58 & $\mathrm{mg} / \mathrm{kg}$ & 3.2 & 2.9 & 3.2 & 0.069 & 8.9 & 0.84 & 5.5 & 1.9 & 4.1 & 1.9 & 0.25 & 58.4 & 0.71 & 0.75 & -0.45 & 0.53 \\
\hline $\mathrm{Cu}$ & 62 & $\mathrm{mg} / \mathrm{kg}$ & 35 & 33 & 34 & 11 & 75 & 21 & 50 & 25 & 44 & 13 & 1.7 & 37.9 & 0.56 & 0.34 & -0.30 & -0.05 \\
\hline Dy & 58 & $\mathrm{mg} / \mathrm{kg}$ & 1.6 & 1.1 & 1.1 & 0.091 & 6.7 & 0.23 & 4.7 & 0.60 & 2.1 & 1.6 & 0.21 & 99.4 & 1.78 & 2.62 & 0.21 & -0.24 \\
\hline $\mathrm{Er}$ & 58 & $\mathrm{mg} / \mathrm{kg}$ & 0.84 & 0.57 & 0.52 & 0.046 & 3.8 & 0.11 & 2.3 & 0.30 & 1.0 & 0.86 & 0.11 & 103 & 1.81 & 2.77 & 0.22 & -0.24 \\
\hline $\mathrm{Eu}$ & 58 & $\mathrm{mg} / \mathrm{kg}$ & 0.52 & 0.37 & 0.35 & 0.025 & 2.2 & 0.10 & 1.2 & 0.18 & 0.65 & 0.49 & 0.065 & 94.5 & 1.73 & 2.64 & 0.13 & -0.19 \\
\hline $\mathrm{Fe}$ & 62 & $\%$ & 4.0 & 3.9 & 3.9 & 2.4 & 6.7 & 3.0 & 5.3 & 3.3 & 4.7 & 0.91 & 0.12 & 22.6 & 0.74 & 0.24 & 0.07 & -0.51 \\
\hline $\mathrm{Ga}$ & 58 & $\mathrm{mg} / \mathrm{kg}$ & 13 & 12 & 13 & 2.8 & 30 & 6.7 & 20 & 9.5 & 16 & 5.2 & 0.69 & 40.1 & 0.76 & 1.40 & -0.25 & 0.72 \\
\hline $\mathrm{Gd}$ & 58 & $\mathrm{mg} / \mathrm{kg}$ & 2.3 & 1.6 & 1.5 & 0.11 & 9.6 & 0.31 & 5.1 & 0.70 & 2.8 & 2.3 & 0.30 & 98.6 & 1.79 & 2.75 & 0.19 & -0.28 \\
\hline $\mathrm{Ge}$ & 58 & $\mathrm{mg} / \mathrm{kg}$ & 0.37 & 0.23 & 0.20 & 0.075 & 1.5 & 0.096 & 1.1 & 0.15 & 0.48 & 0.35 & 0.047 & 96.2 & 1.80 & 2.64 & 0.03 & -1.03 \\
\hline $\mathrm{Hf}$ & 58 & $\mathrm{mg} / \mathrm{kg}$ & 1.1 & 0.92 & 0.94 & 0.071 & 3.0 & 0.31 & 2.7 & 0.43 & 1.6 & 0.82 & 0.11 & 73.8 & 0.84 & -0.31 & 0.04 & -0.79 \\
\hline Ho & 58 & $\mathrm{mg} / \mathrm{kg}$ & 0.31 & 0.21 & 0.21 & 0.018 & 1.4 & 0.041 & 0.88 & 0.12 & 0.37 & 0.32 & 0.041 & 102 & 1.79 & 2.63 & 0.25 & -0.26 \\
\hline I & 58 & $\mu \mathrm{g} / \mathrm{kg}$ & 110 & 36 & 42 & 5.0 & 1500 & 5.0 & 290 & 14 & 95 & 230 & 30 & 209 & 4.84 & 27.93 & 0.03 & -0.66 \\
\hline
\end{tabular}


Table 2 (continuation)

\begin{tabular}{|c|c|c|c|c|c|c|c|c|c|c|c|c|c|c|c|c|c|c|}
\hline In & 58 & $\mathrm{~g} / \mathrm{kg}$ & 37 & 34 & 36 & 5.0 & 96 & 15 & 59 & & 47 & 10 & 2.4 & 49.1 & & 1.26 & -0.06 & \\
\hline K & 2 & & 1.4 & 1.4 & 1.5 & 0.16 & 2.4 & 0.73 & 2.1 & 1.0 & 1.9 & 0.51 & 0.064 & 35.9 & -0.13 & -0.55 & -0.21 & -0.45 \\
\hline $\mathrm{La}$ & 58 & $\mathrm{~g} / \mathrm{kg}$ & 12 & 7.8 & 6.8 & 0.39 & 60 & 1.4 & 30 & 3.5 & 17 & 13 & 1.7 & 105 & 1.66 & 2.71 & 0.06 & -0.50 \\
\hline $\mathrm{Ji}$ & 62 & $\mathrm{~g} / \mathrm{kg}$ & 34 & 33 & 33 & 7.4 & 67 & 19 & 51 & 26 & 39 & 12 & 1.5 & 35.1 & 0.43 & 0.53 & -0.09 & 051 \\
\hline 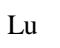 & & 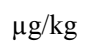 & 120 & & 79 & 5.0 & 520 & 18 & 340 & 46 & 150 & 110 & 15 & 93.8 & & 2.50 & 0.15 & -0.14 \\
\hline & 62 & & & & & 0.21 & 5.8 & & & & & 0.84 & & 91.5 & 3.66 & 18.66 & 0.01 & -0.36 \\
\hline $\mathrm{n}$ & 62 & $\mathrm{ng} / \mathrm{kg}$ & 20 & & 770 & 87 & 4500 & 350 & 1300 & 530 & 1000 & 600 & 76 & 73.0 & 3.92 & 23.58 & 0.24 & 3.00 \\
\hline S & 58 & $\mathrm{ng} / \mathrm{kg}$ & 0.68 & 0.21 & 0.44 & 0.005 & 7.7 & 0.005 & 1.4 & 0.005 & 0.80 & 1.2 & 0.16 & 175 & 4.24 & 22.22 & -0.09 & -1.08 \\
\hline $\mathrm{Na}$ & 62 & & 016 & 0.12 & 0.12 & 0.027 & 0.68 & 0.051 & 0.29 & 071 & 0.20 & 0.12 & 0.015 & 75.8 & 1.98 & 5.64 & -0.04 & -0.35 \\
\hline $\mathrm{Nb}$ & 58 & $\mathrm{ng} / \mathrm{kg}$ & 79 & 22 & 20 & 3.3 & 1300 & 10 & 150 & 12 & 37 & 210 & 27 & 261 & 4.66 & 23.90 & 0.33 & 0.73 \\
\hline $\mathrm{Nd}$ & 58 & $\mathrm{~g} / \mathrm{kg}$ & 9.5 & 7.1 & 6.8 & 0.39 & 39 & 1.4 & 22 & 3.6 & 13 & 8.7 & 1.1 & 91.2 & 1.61 & 2.37 & 0.13 & -0.30 \\
\hline $\mathrm{Ni}$ & 62 & $\mathrm{mg} / \mathrm{kg}$ & 100 & 64 & 68 & 12 & 1200 & 31 & 160 & 43 & 99 & 150 & 20 & 154 & 6.03 & 41.27 & .14 & 1.08 \\
\hline $\mathrm{P}$ & 62 & $\mathrm{~g} / \mathrm{kg}$ & 750 & 650 & 650 & 250 & 2700 & 320 & 1300 & 500 & 890 & 430 & 54 & 57.0 & 1.96 & 6.03 & 0.06 & -0.21 \\
\hline $\mathrm{Pb}$ & 62 & $\mathrm{ng} / \mathrm{kg}$ & 30 & 30 & 29 & 5.0 & 50 & 19 & 43 & & 37 & 9.0 & 1.1 & 29.8 & -0.01 & -0.08 & -0.23 & 0.34 \\
\hline $\mathrm{Pd}$ & 58 & $\mathrm{~g} / \mathrm{kg}$ & 0,1 & 0.34 & 0.36 & 0.050 & 2.0 & 0.050 & 0.95 & 0.15 & 0.61 & 0.39 & 0.052 & 88.1 & 1.80 & 4.25 & 0.07 & -0.41 \\
\hline Pr & 58 & $\mathrm{~g} / \mathrm{kg}$ & 2.4 & 1.8 & 1.7 & 0.098 & 9.9 & 0.35 & 5.6 & 0.98 & & 2.2 & 0.29 & 90.0 & 1.60 & 2.47 & 0.13 & -0.28 \\
\hline $\mathrm{Pt}$ & 58 & $\mathrm{mg} / \mathrm{kg}$ & 0.28 & 0.22 & 0.28 & 0.005 & 1.1 & 0.053 & 0.52 & 0.10 & 0.44 & 0.23 & 0.030 & 80.7 & 1.41 & 3.11 & -0.12 & -0.30 \\
\hline $\mathrm{Ph}$ & 58 & & 45 & 41 & 40 & 0.95 & 135 & 12 & 79 & 28 & 57 & 26 & 3.4 & & & 1.53 & -0.36 & 0.65 \\
\hline $\mathrm{Sb}$ & 58 & $\mathrm{~g} / \mathrm{kg}$ & 1.1 & 51 & 0.59 & 0.067 & 26 & 0.17 & 1.1 & 0.37 & 0.82 & 3.4 & 0.45 & 310 & & 54.95 & -0.02 & 2.69 \\
\hline $\mathrm{Sc}$ & 58 & $\mathrm{~g} / \mathrm{kg}$ & 6.3 & 5.2 & 5.1 & & 25 & 2.4 & 13 & 3.5 & & 4.1 & 0.54 & 66.0 & 1.90 & 5.70 & -0.02 & -0.15 \\
\hline $\mathrm{Sm}$ & 58 & $\mathrm{mg} / \mathrm{kg}$ & 2.0 & 1.4 & 1.3 & 0.050 & 7.8 & 0.32 & 4.4 & 0.67 & 2.6 & 1.8 & 0.24 & 93.6 & 1.6 & 2.36 & 0.12 & -0.22 \\
\hline $\mathrm{Sn}$ & 58 & 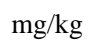 & 15 & $0=$ & 8.5 & 0.67 & 107 & 2.5 & 42 & 3.9 & 19 & 18 & 2.3 & 118 & 2.88 & 11.57 & 0.19 & -0.14 \\
\hline $\mathrm{Sr}$ & 58 & $\mathrm{ng} / \mathrm{kg}$ & 27 & & 21 & & 107 & & 51 & 13 & & 20 & & 75.2 & & 3.85 & & 025 \\
\hline $\mathrm{Ta}$ & 58 & & & & & 050 & 131 & & 1.8 & & & 17 & & 535 & & 57.62 & & 4.49 \\
\hline $\mathrm{Tb}$ & 58 & $\mathrm{ng} / \mathrm{kg}$ & .30 & 22 & 0.22 & 0.017 & 1.2 & 0.043 & 0.76 & 0.11 & 0.40 & 0.30 & 0.039 & 98.1 & 1.78 & 2.64 & 0.26 & -0.26 \\
\hline $\mathrm{Te}$ & 58 & $\mu \mathrm{g} / \mathrm{kg}$ & 36 & 14 & 18 & 5.0 & 290 & 5.0 & 95 & 5.0 & 46 & 49 & 6.5 & 138 & 2.97 & 11.77 & 0.04 & -1.64 \\
\hline $\mathrm{Ti}$ & 58 & & & & 0.29 & 0.11 & 0.74 & & 0.44 & 0.23 & 0.37 & 0.11 & 0.014 & 34.9 & & 3.54 & -0.24 & 0.85 \\
\hline $\mathrm{Tl}$ & Jo & $\mathrm{mg} / \mathrm{kg}$ & 0.47 & 50 & 0.40 & (1) & 1.5 & 0.050 & 0.96 & 0.16 & 0.70 & 0.38 & 0.050 & & & 0.35 & & -1.01 \\
\hline $\mathrm{Tm}$ & 58 & $\mu \mathrm{g} / \mathrm{kg}$ & & & 75 & & 540 & 16 & 320 & 46 & & 120 & 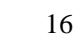 & 101 & & 2.81 & 0.09 & -0.08 \\
\hline V & 62 & $\mathrm{mg} / \mathrm{kg}$ & 120 & 110 & 110 & & 390 & 81 & 170 & 90 & 130 & 49 & .2 & 42.1 & 3.37 & 15.88 & 0.07 & 0.21 \\
\hline W & 58 & $\mathrm{mg} / \mathrm{kg}$ & 1.4 & 1.1 & 1.1 & 0.060 & 5.5 & 0.32 & 3.2 & 0.54 & 1 & 1.1 & 0.15 & 80.7 & 1.32 & 1.87 & 0.06 & -0.40 \\
\hline Y & 58 & $\mathrm{mg} / \mathrm{kg}$ & & & 4.6 & 0.33 & 33 & & 20 & & 12 & 7.9 & & 99.4 & 1.61 & 2.14 & 0.13 & -0.45 \\
\hline $\mathrm{Yb}$ & 58 & $\mathrm{mg} / \mathrm{kg}$ & 0.76 & 53 & 0.47 & 0.036 & 3.4 & 0.098 & 2.1 & 0.30 & 0.94 & 0.75 & 0.099 & 99.4 & 1.77 & 2.74 & 0.17 & -0.21 \\
\hline $\mathrm{Zn}$ & 62 & $\mathrm{mg} / \mathrm{kg}$ & 140 & 130 & 130 & 68 & 350 & 94 & 210 & 110 & 170 & 52 & 6.5 & 36.3 & 1.58 & 3.59 & 0.24 & 0.18 \\
\hline $\mathrm{Zr}$ & 58 & $\mathrm{mg} / \mathrm{kg}$ & 62 & 55 & 51 & 11 & 157 & 22 & 120 & 35 & 97 & 36 & 4.7 & 58.2 & 0.63 & -0.61 & 0.06 & -0.90 \\
\hline
\end{tabular}

$\mathrm{N}$ - Number of samples; X - mean; X(BC) - mean of Box-Cox transformed values; Md - median; Min - minimum;

Max - maximum; $\mathrm{P}_{10}-10^{\text {th }}$ percentile; $\mathrm{P}_{25}-25^{\text {th }}$ percentile; $\mathrm{P}_{75}-75^{\text {th }}$ percentile $; \mathrm{P}_{90}-90^{\text {th }}$ percentile; $\mathrm{S}-$ standard deviation; $\mathrm{S}_{\mathrm{x}}$ - standard deviation of transformed values; CV - coefficient of variation, A - skewness; E - kurtosis;

$\mathrm{BC}-$ Box-Cox transformed values 
Table 3. The ratios of the element average content in topsoil (TS) and subsoil (SS) samples from the Crn Drim River Basin

\begin{tabular}{|c|c|c|c|c|c|c|c|c|c|}
\hline Element & Topsoil & Subsoil & $\begin{array}{c}\text { Ratio } \\
\text { (TS/SS) }\end{array}$ & $\mathrm{T}$ (test) & Sign & F (ratio) & Sign & $\mathrm{R}(\mathrm{TS} / \mathrm{SS})$ & Sign \\
\hline $\mathrm{Ag}$ & 0.76 & 0.80 & 0.95 & -1.35 & NS & 1.07 & NS & 0.45 & $*$ \\
\hline $\mathrm{Al}$ & 17900 & 20100 & 0.89 & -1.09 & NS & 1.11 & NS & 0.51 & * \\
\hline As & 16.23 & 16.44 & 0.99 & -0.10 & NS & 1.08 & NS & 0.30 & $*$ \\
\hline B & 54.8 & 56.3 & 0.97 & -0.46 & NS & 1.09 & NS & 0.52 & $*$ \\
\hline $\mathrm{Ba}$ & 274 & 256 & 1.07 & 0.60 & NS & 1.44 & NS & 0.86 & $*$ \\
\hline $\mathrm{Be}$ & 2.18 & 2.25 & 0.97 & -0.31 & NS & 1.11 & NS & 0.58 & $*$ \\
\hline $\mathrm{Bi}$ & 0.18 & 0.16 & 1.14 & 0.79 & NS & 1.24 & NS & 0.25 & NS \\
\hline $\mathrm{Br}$ & 2.69 & 2.96 & 0.91 & -0.56 & NS & 1.72 & NS & 0.23 & NS \\
\hline $\mathrm{Ca}$ & 54799 & 5560 & 0.99 & -0.10 & NS & 1.71 & NS & 0.79 & $*$ \\
\hline $\mathrm{Cd}$ & 0.30 & 0.34 & 0.88 & -0.47 & NS & 1.13 & NS & 0.64 & $*$ \\
\hline $\mathrm{Ce}$ & 15.52 & 14.82 & 1.05 & 0.25 & NS & 1.39 & NS & 0.40 & $*$ \\
\hline Co & 14.23 & 14.15 & 1.01 & 0.04 & NS & 1.26 & NS & 0.54 & $*$ \\
\hline $\mathrm{Cr}$ & 85.0 & 86.2 & 0.99 & -0.20 & NS & 1.05 & NS & 0.90 & $*$ \\
\hline Cs & 3.14 & 2.89 & 1.09 & 0.63 & NS & 1.15 & NS & 0.48 & $*$ \\
\hline $\mathrm{Cu}$ & 30.95 & 33.05 & 0.94 & -0.86 & NS & 1.22 & NS & 0.76 & $*$ \\
\hline Dy & 1.13 & 1.14 & 1.00 & -0.02 & NS & 1.00 & NS & 0.40 & $*$ \\
\hline $\mathrm{Er}$ & 0.56 & 0.57 & 0.99 & -0.07 & NS & 1.03 & NS & 0.43 & $*$ \\
\hline $\mathrm{Eu}$ & 0.40 & 0.37 & 1.07 & 0.37 & NS & 1.08 & NS & 0.31 & $*$ \\
\hline $\mathrm{Fe}$ & 36932 & 38931 & 0.95 & -1.32 & NS & 1.15 & NS & 0.87 & $*$ \\
\hline $\mathrm{Ga}$ & 12.11 & 12.32 & 0.98 & -0.21 & NS & 1.20 & NS & 0.21 & NS \\
\hline Gd & 1.64 & 1.60 & 1.03 & 0.14 & NS & 1.08 & NS & 0.36 & $*$ \\
\hline $\mathrm{Ge}$ & 0.22 & 0.23 & 0.96 & -0.27 & NS & 1.10 & NS & 0.00 & NS \\
\hline $\mathrm{Hf}$ & 0.90 & 0.92 & 0.98 & -0.12 & NS & 1.10 & NS & 0.20 & NS \\
\hline Ho & 0.21 & 0.21 & 0.98 & -0.08 & NS & 1.04 & NS & 0.43 & $*$ \\
\hline I & 0.02 & 0.04 & 0.63 & -1.72 & NS & 1.00 & NS & 0.35 & $*$ \\
\hline In & 0.03 & 0.03 & 0.99 & -0.08 & NS & 1.27 & NS & 0.08 & NS \\
\hline K & 13792 & 14033 & 0.98 & -0.27 & NS & 1.04 & NS & 0.91 & $*$ \\
\hline $\mathrm{La}$ & 8.40 & 7.75 & 1.08 & 0.40 & NS & 1.34 & NS & 0.46 & $*$ \\
\hline $\mathrm{Li}$ & 31.18 & 32.91 & 0.95 & -0.83 & NS & 1.09 & NS & 0.93 & $*$ \\
\hline $\mathrm{Lu}$ & 0.09 & 0.09 & 1.00 & 0.00 & NS & 1.07 & NS & 0.33 & $*$ \\
\hline $\mathrm{Mg}$ & 6722 & 6701 & 1.00 & 0.03 & NS & 1.07 & NS & 0.88 & $*$ \\
\hline Mn & 732 & 715.6 & 1.02 & 0.20 & NS & 1.18 & NS & 0.93 & $*$ \\
\hline Mo & 0.24 & 0.21 & 1.18 & 0.42 & NS & 1.02 & NS & 0.21 & NS \\
\hline $\mathrm{Na}$ & 1328 & 1205 & 1.10 & 0.82 & NS & 1.31 & NS & 0.91 & $*$ \\
\hline $\mathrm{Nb}$ & 19.4 & 22.1 & 0.88 & -0.69 & NS & 1.28 & NS & 0.62 & $*$ \\
\hline $\mathrm{Nd}$ & 7.48 & 7.07 & 1.06 & 0.33 & NS & 1.24 & NS & 0.36 & $*$ \\
\hline $\mathrm{Ni}$ & 62.6 & 64.2 & 0.97 & -0.20 & NS & 1.15 & NS & 0.93 & $*$ \\
\hline $\mathrm{P}$ & 720 & 648 & 1.11 & 1.20 & NS & 1.24 & NS & 0.90 & $*$ \\
\hline $\mathrm{Pb}$ & 32.24 & 30.01 & 1.07 & 1.35 & NS & 1.00 & NS & 0.73 & $*$ \\
\hline $\mathrm{Pd}$ & 0.41 & 0.34 & 1.21 & 1.13 & NS & 1.17 & NS & 0.24 & NS \\
\hline $\operatorname{Pr}$ & 1.94 & 1.81 & 1.07 & 0.39 & NS & 1.28 & NS & 0.36 & $*$ \\
\hline $\mathrm{Pt}$ & 0.24 & 0.22 & 1.09 & 0.46 & NS & 1.21 & NS & 0.02 & NS \\
\hline $\mathrm{Rb}$ & 46.83 & 40.59 & 1.15 & 1.14 & NS & 1.21 & NS & 0.42 & $*$ \\
\hline $\mathrm{Sb}$ & 0.57 & 0.51 & 1.11 & 0.67 & NS & 1.05 & NS & 0.66 & $*$ \\
\hline $\mathrm{Sc}$ & 5.09 & 5.20 & 0.98 & -0.17 & NS & 1.12 & NS & 0.28 & NS \\
\hline $\mathrm{Sm}$ & 1.47 & 1.42 & 1.04 & 0.22 & NS & 1.20 & NS & 0.35 & $*$ \\
\hline $\mathrm{Sn}$ & 10.75 & 9.48 & 1.13 & 0.59 & NS & 1.13 & NS & 0.17 & NS \\
\hline $\mathrm{Sr}$ & 25.03 & 22.97 & 1.09 & 0.64 & NS & 1.14 & NS & 0.55 & $*$ \\
\hline Ta & 0.62 & 0.71 & 0.88 & -0.65 & NS & 1.01 & NS & 0.45 & $*$ \\
\hline $\mathrm{Tb}$ & 0.22 & 0.22 & 1.01 & 0.03 & NS & 1.01 & NS & 0.37 & $*$ \\
\hline
\end{tabular}




\begin{tabular}{lccccccccc}
\multicolumn{2}{l}{ Table 3 (continuation) } & & & & & & \\
$\mathrm{Te}$ & 0.01 & 0.01 & 0.79 & -1.22 & $\mathrm{NS}$ & 1.07 & $\mathrm{NS}$ & 0.66 & $*$ \\
$\mathrm{Ti}$ & 2921 & 2906 & 1.01 & 0.07 & $\mathrm{NS}$ & 1.43 & $\mathrm{NS}$ & 0.22 & NS \\
$\mathrm{Tl}$ & 0.36 & 0.36 & 0.99 & -0.07 & $\mathrm{NS}$ & 1.06 & $\mathrm{NS}$ & 0.18 & NS \\
$\mathrm{Tm}$ & 0.08 & 0.08 & 1.01 & 0.03 & $\mathrm{NS}$ & 1.06 & $\mathrm{NS}$ & 0.42 & $*$ \\
$\mathrm{~V}$ & 99.1 & 105 & 0.94 & -1.26 & $\mathrm{NS}$ & 1.16 & $\mathrm{NS}$ & 0.90 & $*$ \\
$\mathrm{~W}$ & 1.14 & 1.11 & 1.03 & 0.16 & $\mathrm{NS}$ & 1.02 & $\mathrm{NS}$ & 0.26 & $\mathrm{NS}$ \\
$\mathrm{Y}$ & 5.34 & 5.38 & 0.99 & -0.04 & $\mathrm{NS}$ & 1.26 & $\mathrm{NS}$ & 0.61 & $*$ \\
$\mathrm{Yb}$ & 0.52 & 0.53 & 0.98 & -0.09 & $\mathrm{NS}$ & 1.00 & $\mathrm{NS}$ & 0.40 & $*$ \\
$\mathrm{Zn}$ & 132.8 & 132.6 & 1.00 & 0.03 & $\mathrm{NS}$ & 1.16 & $\mathrm{NS}$ & 0.65 & $*$ \\
$\mathrm{Zr}$ & 52.82 & 55.22 & 0.96 & -0.40 & $\mathrm{NS}$ & 1.25 & $\mathrm{NS}$ & 0.51 & $*$ \\
\hline
\end{tabular}

NS - non-significant; * - significant

In order to determine the dependence of the average contents (Box Cox) of the analysed elements between the topsoil and the subsoil, the ratio of the contents was calculated (Table 3). The elements distribution should not vary significantly between the topsoil $(0-5 \mathrm{~cm})$ and the subsoil $(20-30$ $\mathrm{cm}$ ), except if certain destructive anthropogenic or natural processes contribute to variation in the concentration. For almost all elements, insignificant differences were noted for their content in the topsoil versus subsoil. Thus, this relation varied from 0.88 for $\mathrm{Cd}$ and Ta to 1.18 for Mo, which shows an absence of a significant influence of possible soil pollution from anthropogenic activities. It should be noted that the significant difference in iodine content (ration of 0.63 ) is due to iodine dynamics in soils, by its movement beyond the topsoil during rainfall or drainage events; it appears to be effectively retained in the deeper soil horizons by the substantial adsorption capacity provided by relatively small amounts of humus [33].

A comparative analysis conducted based on the contents of the analysed elements in topsoils from the Crn Drim River Basin region and soil from Macedonia [20] and Europe [29] is given in Table 4. For the comparative analysis, median values were used as a more stable parameter, as well as the range of the content for each element. The median content of $\mathrm{Al}(1.7 \%)$ and $\mathrm{Fe}(3.6 \%)$ was higher than the median for the soil from the whole territory of Macedonia (1.3\% and $3.5 \%$, respectively), while the contents of the other macro-elements $(\mathrm{Ca}, \mathrm{Fe}, \mathrm{K}, \mathrm{Li}$, $\mathrm{Mg}, \mathrm{Na}$ and $\mathrm{Ti}$ ) were lower in relation to the Macedonian soil. The median values for some of the trace elements were lower (Ba, Bi, Ce, Co, $\mathrm{La}, \mathrm{Mo}, \mathrm{Rb}$, $\mathrm{Sb}, \mathrm{Sc}, \mathrm{Tl}, \mathrm{W}$ and $\mathrm{Y}$ ), higher for some elements (As, $\mathrm{Be}, \mathrm{Nb}, \mathrm{Ni}, \mathrm{P}, \mathrm{Sn}, \mathrm{Sr}$, Ta and V) and very similar for other elements $(\mathrm{Cd}, \mathrm{Cr}, \mathrm{Cu}, \mathrm{Hf}, \mathrm{Li}, \mathrm{Mn}, \mathrm{Pb})$ in soil samples from Crn Drim River Basin compared with those from Macedonian soil, which shows that their distribution corresponds to the lithogenic origin of the rocks. Comparing the median values of soil from Crn Drim River Basin with the European soil (Table 4), the content of $\mathrm{Al}$ and $\mathrm{Na}$ was much lower in relation to the data published by Salminen et al. [30], while for the other macro-elements the values did not show significant variations. The distribution of the remaining chemical elements corresponded to the lithogenic origin of the rocks in the separate subregions of the area (Figure 3). The only major difference was in the content of $\mathrm{Cu}$ and $\mathrm{Zn}$, which were more than two times higher in topsoil from the Crn Drim River Basin than in the European soil. It is important to note that, except in the case of $\mathrm{Ba}$, the median value for all the elements included in The New Dutchlist, such as As, Cd, Co, Cr, Cu, Pb, Mo, $\mathrm{Ni}$ and $\mathrm{Zn}$ (http://www.contaminatedland.co.uk), were mostly below the target values.

Because of the great number of variables, data reduction was performed using factor analysis; this was performed only for the elements analysed by ICP-AES. A matrix of correlation coefficients was produced based on previously standardised and Box-Cox transformed values for element contents in the samples of topsoil and subsoil (Table 5). In the factor analysis, 62 samples of topsoil $(0-5 \mathrm{~cm})$ and 62 samples of subsoil $(20-30 \mathrm{~cm})$ and the analysis of 19 chemical elements were considered. From the R-mode factor analysis, three chemical elements (B, $\mathrm{Li}$ and $\mathrm{P}$ ) were eliminated from further analysis because they had low shares of communality or low tendencies to form independent factors. Table 5 shows the loadings of values for each individual element on each factor, showing four geochemical associations. The total communality of the factors was $69.8 \%$ (Table 5).

Based on the results of the factor analysis (Table 5) and the trends shown on the geochemical maps, four natural geochemical associations in the soil have been defined: Factor $1(\mathrm{Ba}, \mathrm{K}, \mathrm{Ni})$, Factor $2(\mathrm{Ag}, \mathrm{Cd}, \mathrm{Cu}, \mathrm{Pb}, \mathrm{Zn})$, Factor $3(\mathrm{Cr}, \mathrm{Fe}, \mathrm{Na}, \mathrm{Ni}, \mathrm{V})$ and Factor 4 (Al, Ca, Mg, Mn). 
Table 4. Comparison of the median, minimal and maximal values of the content of the analysed elements in top-soil from the Crn Drim River Basin with soil from Macedonia and Europe

\begin{tabular}{|c|c|c|c|c|c|c|c|c|c|}
\hline \multirow[t]{2}{*}{ Element } & \multirow[t]{2}{*}{ Unit } & \multicolumn{2}{|c|}{ Dutchlist } & \multicolumn{2}{|c|}{ Crn Drim River Basin } & \multicolumn{2}{|c|}{ Macedonia [20] } & \multicolumn{2}{|c|}{ Europe [30] } \\
\hline & & Target & Action & $\mathrm{Md}$ & Min-Max & $\mathrm{Md}$ & Min-Max & $\mathrm{Md}$ & Min-Max \\
\hline $\mathrm{Ag}$ & $\mathrm{mg} / \mathrm{kg}$ & & & 0.75 & $0.48-1.2$ & - & - & 0.27 & $0.01-3.15$ \\
\hline $\mathrm{Al}$ & $\%$ & & & 1.7 & $0.44-6.5$ & 1.3 & $0.05-35$ & 5.8 & $0.70-14.1$ \\
\hline As & $\mathrm{mg} / \mathrm{kg}$ & 29 & 55 & 16 & $4.7-270$ & 10 & $1.0-720$ & 12 & $0.32-562$ \\
\hline $\mathrm{B}$ & $\mathrm{mg} / \mathrm{kg}$ & & & 54 & $26-114$ & - & - & - & - \\
\hline $\mathrm{Ba}$ & $\mathrm{mg} / \mathrm{kg}$ & 200 & 625 & 250 & $65-940$ & 430 & $6-2900$ & 375 & $30-1870$ \\
\hline $\mathrm{Be}$ & $\mathrm{mg} / \mathrm{kg}$ & & & 2.3 & $0.072-4.9$ & 2.0 & $<1.0-8.0$ & $<2.0$ & $<2.0-18.7$ \\
\hline $\mathrm{Bi}$ & $\mathrm{mg} / \mathrm{kg}$ & & & 0.18 & $0.005-0.83$ & 0.30 & $<0.1-15$ & $<0.5$ & $<0.5-9.57$ \\
\hline $\mathrm{Br}$ & $\mathrm{mg} / \mathrm{kg}$ & & & 2.9 & $0.005-6.9$ & - & - & - & - \\
\hline $\mathrm{Ca}$ & $\%$ & & & 0.56 & $0.11-4.6$ & 1.3 & $0.05-35$ & 0.66 & $0.019-34.3$ \\
\hline $\mathrm{Cd}$ & $\mathrm{mg} / \mathrm{kg}$ & 0.8 & 12 & 0.30 & $0.005-3.4$ & 0.30 & $0.01-110$ & 0.92 & 0.03-14 \\
\hline $\mathrm{Ce}$ & $\mathrm{mg} / \mathrm{kg}$ & & & 16 & $1.3-58$ & 56 & $1.0-180$ & 48.2 & $1.04-379$ \\
\hline Co & $\mathrm{mg} / \mathrm{kg}$ & 20 & 240 & 13 & $1.0-76$ & 17 & $0.50-150$ & 8.0 & $<1.0-191$ \\
\hline $\mathrm{Cr}$ & $\mathrm{mg} / \mathrm{kg}$ & 100 & 380 & 87 & $50-1000$ & 88 & $5.0-2700$ & 60 & $<3-6230$ \\
\hline Cs & $\mathrm{mg} / \mathrm{kg}$ & & & 3.1 & $0.24-11$ & - & - & 2.71 & $<0.5-69$ \\
\hline $\mathrm{Cu}$ & $\mathrm{mg} / \mathrm{kg}$ & 36 & 190 & 30 & $8.0-125$ & 28 & $1.6-270$ & 13 & $0.81-256$ \\
\hline Dy & $\mathrm{mg} / \mathrm{kg}$ & & & 1.5 & $0.037-6.9$ & - & - & 3.42 & $0.18-45$ \\
\hline $\mathrm{Er}$ & $\mathrm{mg} / \mathrm{kg}$ & & & 0.71 & $0.016-3.5$ & - & - & 1.98 & $0.12-26$ \\
\hline $\mathrm{Eu}$ & $\mathrm{mg} / \mathrm{kg}$ & & & 0.40 & $0.051-1.7$ & - & - & 0.77 & $0.05-7.0$ \\
\hline $\mathrm{Fe}$ & $\%$ & & & 3.6 & $2.2-6.6$ & 3.5 & $0.03-12$ & 1.34 & $0.049-10.6$ \\
\hline $\mathrm{Ga}$ & $\mathrm{mg} / \mathrm{kg}$ & & & 12 & $3.5-37$ & - & - & 13.5 & $0.54-34$ \\
\hline $\mathrm{Gd}$ & $\mathrm{mg} / \mathrm{kg}$ & & & 1.9 & $0.077-7.6$ & - & - & 3.85 & $0.20-36$ \\
\hline $\mathrm{Ge}$ & $\mathrm{mg} / \mathrm{kg}$ & & & 0.20 & $0.070-2.4$ & - & - & - & - \\
\hline Hf & $\mathrm{mg} / \mathrm{kg}$ & & & 1.0 & $0.065-3.4$ & 1.0 & $<0.10-6.6$ & 5.55 & $<0.2-21$ \\
\hline Ho & $\mathrm{mg} / \mathrm{kg}$ & & & 0.26 & $0.005-1.2$ & - & - & 0.72 & $0.03-9.2$ \\
\hline I & $\mathrm{mg} / \mathrm{kg}$ & & & 22 & $5.0-250$ & - & - & 3.94 & $<2-71$ \\
\hline In & $\mathrm{mg} / \mathrm{kg}$ & & & 0.036 & $0.005-0.11$ & - & - & 0.05 & $<0.01-0.41$ \\
\hline $\mathrm{K}$ & $\%$ & & & 1.3 & $0.33-2.4$ & 1.9 & $0.02-5.3$ & 1.59 & $0.022-5.1$ \\
\hline $\mathrm{La}$ & $\mathrm{mg} / \mathrm{kg}$ & & & 9.8 & $0.59-57$ & 25 & $0.60-88$ & 23 & $1.10-143$ \\
\hline $\mathrm{Li}$ & $\mathrm{mg} / \mathrm{kg}$ & & & 30 & $12-62$ & 26 & $1.8-210$ & - & - \\
\hline $\mathrm{Lu}$ & $\mathrm{mg} / \mathrm{kg}$ & & & 0.11 & $0.005-0.46$ & - & - & 0.30 & $<0.02-3.21$ \\
\hline $\mathrm{Mg}$ & $\%$ & & & 0.64 & $0.22-7.2$ & 0.94 & $0.12-13$ & 0.47 & $<0.006-15$ \\
\hline $\mathrm{Mn}$ & $\mathrm{mg} / \mathrm{kg}$ & & & 820 & $61-3700$ & 900 & $17-10000$ & 510 & $31-6070$ \\
\hline Mo & $\mathrm{mg} / \mathrm{kg}$ & 10 & 200 & 0.43 & $0.005-3.0$ & 0.90 & $<0.10-51$ & 0.61 & $<0.10-21$ \\
\hline $\mathrm{Na}$ & $\%$ & & & 0.12 & $0.029-0.67$ & 1.3 & $0.007-3.7$ & 0.6 & $0.03-3.34$ \\
\hline $\mathrm{Nb}$ & $\mathrm{mg} / \mathrm{kg}$ & & & 19 & $3.0-950$ & 11 & $0.30-2000$ & 9.68 & $0.45-134$ \\
\hline $\mathrm{Nd}$ & $\mathrm{mg} / \mathrm{kg}$ & & & 8.3 & $0.54-29$ & - & - & 21 & $1.14-132$ \\
\hline $\mathrm{Ni}$ & $\mathrm{mg} / \mathrm{kg}$ & 35 & 210 & 61 & $12-1000$ & 46 & $2.1-2500$ & 18 & $<2-2690$ \\
\hline $\mathrm{P}$ & $\mathrm{mg} / \mathrm{kg}$ & & & 730 & $250-2600$ & 620 & $110-3900$ & 960 & $82-9900$ \\
\hline $\mathrm{Pb}$ & $\mathrm{mg} / \mathrm{kg}$ & 85 & 530 & 31 & $14-62$ & 32 & $1.2-10000$ & 23 & $5.3-970$ \\
\hline $\mathrm{Pd}$ & $\mathrm{mg} / \mathrm{kg}$ & & & 0.41 & $0.050-1.7$ & - & - & - & - \\
\hline $\operatorname{Pr}$ & $\mathrm{mg} / \mathrm{kg}$ & & & 2.2 & $0.14-7.0$ & - & - & 5.6 & $0.29-31.6$ \\
\hline $\mathrm{Pt}$ & $\mathrm{mg} / \mathrm{kg}$ & & & 0.30 & $0.005-1.0$ & - & - & - & - \\
\hline $\mathrm{Rb}$ & $\mathrm{mg} / \mathrm{kg}$ & & & 46 & $3.5-148$ & 86 & $0.70-390$ & - & - \\
\hline $\mathrm{Sb}$ & $\mathrm{mg} / \mathrm{kg}$ & & & 0.64 & $0.074-45$ & 0.80 & $<0.10-630$ & 0.60 & $0.02-31$ \\
\hline $\mathrm{Sc}$ & $\mathrm{mg} / \mathrm{kg}$ & & & 5.5 & $1.1-33$ & 12 & $<1.0-39$ & 8.21 & $<0.50-54$ \\
\hline $\mathrm{Sm}$ & $\mathrm{mg} / \mathrm{kg}$ & & & 1.7 & $0.10-6.1$ & - & - & 3.96 & $0.23-30$ \\
\hline $\mathrm{Sn}$ & $\mathrm{mg} / \mathrm{kg}$ & & & 10 & $0.30-141$ & 2.6 & $<0.10-680$ & 3.00 & $<2.0-106$ \\
\hline $\mathrm{Sr}$ & $\mathrm{mg} / \mathrm{kg}$ & & & 27 & $0.88-79$ & 140 & $21-1400$ & 89 & $8-3120$ \\
\hline $\mathrm{Ta}$ & $\mathrm{mg} / \mathrm{kg}$ & & & 0.75 & $0.050-7.6$ & 0.70 & $<0.10-30$ & 0.68 & $<0.05-6.8$ \\
\hline $\mathrm{Tb}$ & $\mathrm{mg} / \mathrm{kg}$ & & & 0.26 & $0.005-1.0$ & - & - & 0.60 & $0.03-7.0$ \\
\hline $\mathrm{Te}$ & $\mathrm{mg} / \mathrm{kg}$ & & & 0.005 & $0.005-0.20$ & - & - & 0.03 & $<0.02-0.93$ \\
\hline $\mathrm{Ti}$ & $\%$ & & & 0.29 & $0.10-0.84$ & 0.34 & $0.004-1.2$ & 0.34 & $0.012-3.27$ \\
\hline $\mathrm{Tl}$ & $\mathrm{mg} / \mathrm{kg}$ & & & 0.38 & $0.050-1.5$ & 0.70 & $<0.50-16$ & 0.66 & $0.05-24$ \\
\hline $\mathrm{Tm}$ & $\mathrm{mg} / \mathrm{kg}$ & & & 0.10 & $0.005-0.48$ & - & - & 0.30 & $0.05-4.03$ \\
\hline V & $\mathrm{mg} / \mathrm{kg}$ & & & 98 & $65-410$ & 89 & $1.0-470$ & 60 & $2.7-537$ \\
\hline W & $\mathrm{mg} / \mathrm{kg}$ & & & 1.1 & $0.064-4.8$ & 1.3 & $0.20-18$ & $<5.0$ & $<5.0-14$ \\
\hline $\mathrm{Y}$ & $\mathrm{mg} / \mathrm{kg}$ & & & 4.9 & $0.22-29$ & 18 & $0.30-110$ & 21 & $<3.0-267$ \\
\hline $\mathrm{Yb}$ & $\mathrm{mg} / \mathrm{kg}$ & & & 0.66 & $0.018-3.1$ & - & - & 1.99 & $0.09-25$ \\
\hline $\mathrm{Zn}$ & $\mathrm{mg} / \mathrm{kg}$ & & & 130 & $58-290$ & 83 & $8.0-10000$ & 52 & $<3-2900$ \\
\hline $\mathrm{Zr}$ & $\mathrm{mg} / \mathrm{kg}$ & 140 & 720 & 56 & $7.1-144$ & 35 & $0.80-210$ & 231 & $5.0-1060$ \\
\hline
\end{tabular}

Md - median; Min - minimum; Max - Maximum 
Table 5. Matrix of dominant rotated factor loadings $(\mathrm{n}=124,16$ selected elements)

\begin{tabular}{cccccc}
\hline Element & $\mathrm{F} 1$ & $\mathrm{~F} 2$ & $\mathrm{~F} 3$ & $\mathrm{~F} 4$ & $\mathrm{Comm}$ \\
\hline $\mathrm{Ba}$ & $\mathbf{0 . 8 2}$ & 0.17 & 0.05 & -0.22 & 75.5 \\
$\mathrm{~K}$ & $\mathbf{0 . 8 6}$ & -0.13 & -0.02 & -0.07 & 75.7 \\
$\mathrm{Ag}$ & -0.08 & $\mathbf{0 . 6 7}$ & 0.21 & 0.20 & 54.0 \\
$\mathrm{Cd}$ & -0.34 & $\mathbf{0 . 7 1}$ & -0.01 & 0.01 & 62.4 \\
$\mathrm{Cu}$ & -0.14 & $\mathbf{0 . 5 5}$ & 0.30 & 0.45 & 58.5 \\
$\mathrm{~Pb}$ & 0.33 & $\mathbf{0 . 6 7}$ & -0.25 & 0.00 & 62.1 \\
$\mathrm{Zn}$ & 0.16 & $\mathbf{0 . 7 9}$ & 0.34 & 0.17 & 80.0 \\
$\mathrm{Cr}$ & -0.39 & -0.02 & $\mathbf{0 . 8 2}$ & 0.12 & 82.9 \\
$\mathrm{Fe}$ & -0.09 & 0.20 & $\mathbf{0 . 7 2}$ & 0.38 & 70.2 \\
$\mathrm{Na}$ & 0.15 & -0.17 & $\mathbf{0 . 6 0}$ & 0.38 & 56.0 \\
$\mathrm{Ni}$ & $-\mathbf{0 . 6 0}$ & 0.17 & $\mathbf{- 0 . 6 2}$ & 0.31 & 87.2 \\
$\mathrm{~V}$ & 0.24 & 0.28 & $\mathbf{0 . 7 7}$ & 0.02 & 73.0 \\
$\mathrm{Al}$ & 0.09 & 0.12 & 0.22 & $\mathbf{0 . 8 0}$ & 71.0 \\
$\mathrm{Ca}$ & -0.28 & 0.34 & -0.06 & $\mathbf{0 . 7 2}$ & 72.2 \\
$\mathrm{Mg}$ & -0.19 & -0.12 & 0.29 & $\mathbf{0 . 8 4}$ & 84.1 \\
$\mathrm{Mn}$ & -0.27 & 0.18 & 0.19 & $\mathbf{0 . 6 2}$ & 52.4 \\
Prp. Totl. & 15.6 & 16.6 & 19.1 & 18.6 & 69.8 \\
Eigen Val & 5.52 & 2.42 & 1.90 & 1.33 & \\
Expl. Var, & 2.50 & 2.65 & 3.05 & 2.97 & \\
\hline
\end{tabular}

F1, F2, F3, F4 - Factor loadings; Com - Communality (\%); Var - Variance (\%)

Factor $1(\mathrm{Ba}, \mathrm{K}, \mathrm{Ni})$ is a lithogenic and geogenic association. The spatial distribution of the scores of this factor is given in Figure 5, both for topsoil and bottom soil samples. It is obvious that the highest values for $\mathrm{Ba}$ and $\mathrm{K}$ content were in areas occupied by the Precambrian and Paleozoic schists, and Paleozoic sandstones. From Tables 1 and 2, it can be determined that the Ba content in the topsoil ranged from 65 to $940 \mathrm{mg} / \mathrm{kg}$, while in the bottom soil its values were from 28 to $980 \mathrm{mg} / \mathrm{kg}$. The median in the surface layer was $250 \mathrm{mg} / \mathrm{kg}$, and in the subsoil was $240 \mathrm{mg} / \mathrm{kg}$. The highest contents of this element were observed in soil samples from the Precambrian and Paleozoic schists, represented in the central part of the area; that is, on the border between the municipalities of Ohrid and Struga. The high $\mathrm{Ba}$ content was also found in the soils from the Paleozoic sandstones, represented in the Gorna Debarca area, which is primarily characterised by hilly and mountainous relief. Potassium content ranged from $0.33 \%$ to $2.4 \%$ in the topsoil and from 0.16 to $2.4 \%$ in the subsoil, with median values of $1.3 \%$ and $1.5 \%$, respectively. According to the distribution map, its representation extends to the Precambrian and Paleozoic schists, Paleozoic sandstones, and Paleozoic and Mesozoic carbonates. The correlation for $\mathrm{Ni}$ in this Factor is negative and its spatial distribution was much different than that of the other elements (Figure 6); it is obvious that its content was the lowest in the previously mentioned areas and highest in the areas occupied by Paleozoic and Mesozoic carbonates (Mts. Galičica and Jablanica) and by Tertiary sediments (Figures 3 and 6). Nickel content ranged from 12 to $1000 \mathrm{mg} / \mathrm{kg}$ and from 12 to $1200 \mathrm{mg} / \mathrm{kg}$ in top- and subsoils, with median values of $61 \mathrm{mg} / \mathrm{kg}$ and $65 \mathrm{mg} / \mathrm{kg}$, respectively. 


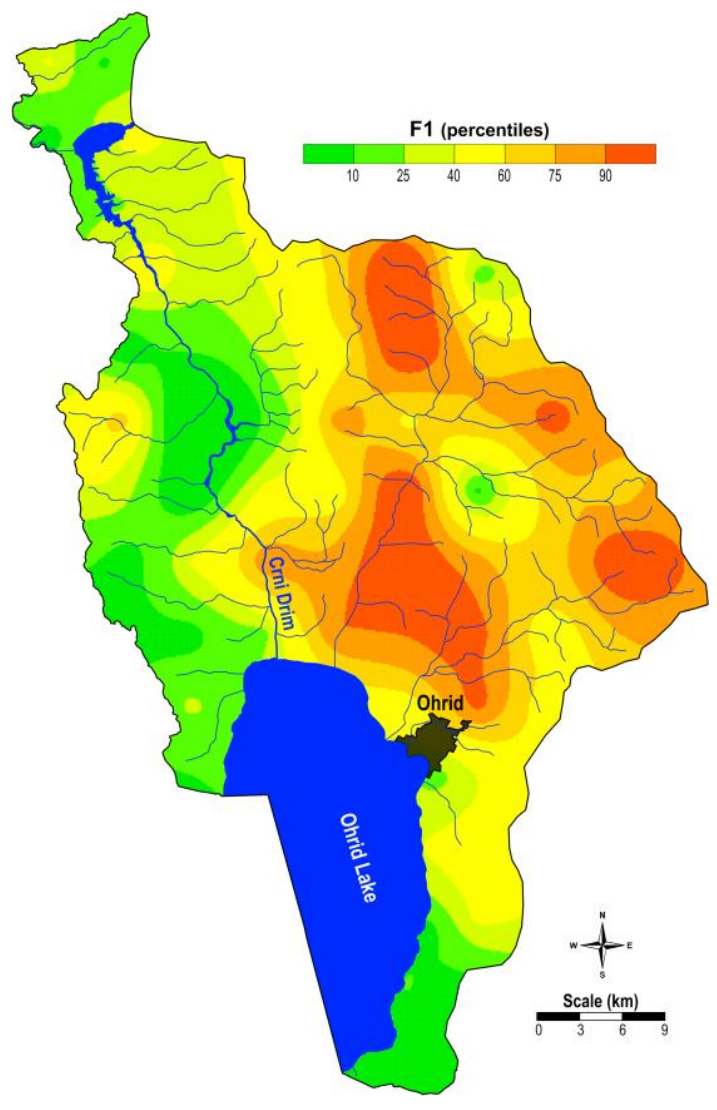

Figure 5. Spatial distribution of factor scores of F1 (Ba, K, Ni) in the Crn Drim River Basin

Factor $2(\mathrm{Ag}, \mathrm{Cd}, \mathrm{Cu}, \mathrm{Pb}, \mathrm{Zn})$ represents the geogenic association. The spatial distributions of the factor scores of this factor for topsoil and bottom soil are shown in Figure 7. The origins of these elements are mostly related to the Paleozoic and Mesozoic carbonates. The highest contents these elements were in the areas of Mts. Galičica and Jablanica.

The content of silver ranged from 0.48 to 1.2 $\mathrm{mg} / \mathrm{kg}$ in both top- and subsoil, with very close median values of $0.75 \mathrm{mg} / \mathrm{kg}$ and $0.80 \mathrm{mg} / \mathrm{kg}$, respectively. From the similarity of these values, it can be concluded that there is no contamination of the surface layer of the soil from anthropogenic sources. The highest contents for silver were found in the Palaeozoic and Mesozoic carbonates, that are noticeable over Mount Galičica and around the city of Ohrid, and in the northeastern part of Gorna Debarca, where many types of rocks are present: Tertiary sediments, Precambrian and Paleozoic schists and magmatic rocks. A similar distribution was also observed for $\mathrm{Cd}$ and $\mathrm{Cu}$. The median value for $\mathrm{Cd}$ was $0.30 \mathrm{mg} / \mathrm{kg}$ in the topsoil, with a range of 0.005 to $3.4 \mathrm{mg} / \mathrm{kg}$, and $0.29 \mathrm{mg} / \mathrm{kg}$ in the subsoil soil with a range of 0.005 to $7.3 \mathrm{mg} / \mathrm{kg}$. The median value for $\mathrm{Cu}$ in the topsoil was $30 \mathrm{mg} / \mathrm{kg}$, which is significantly higher compared to the median of the

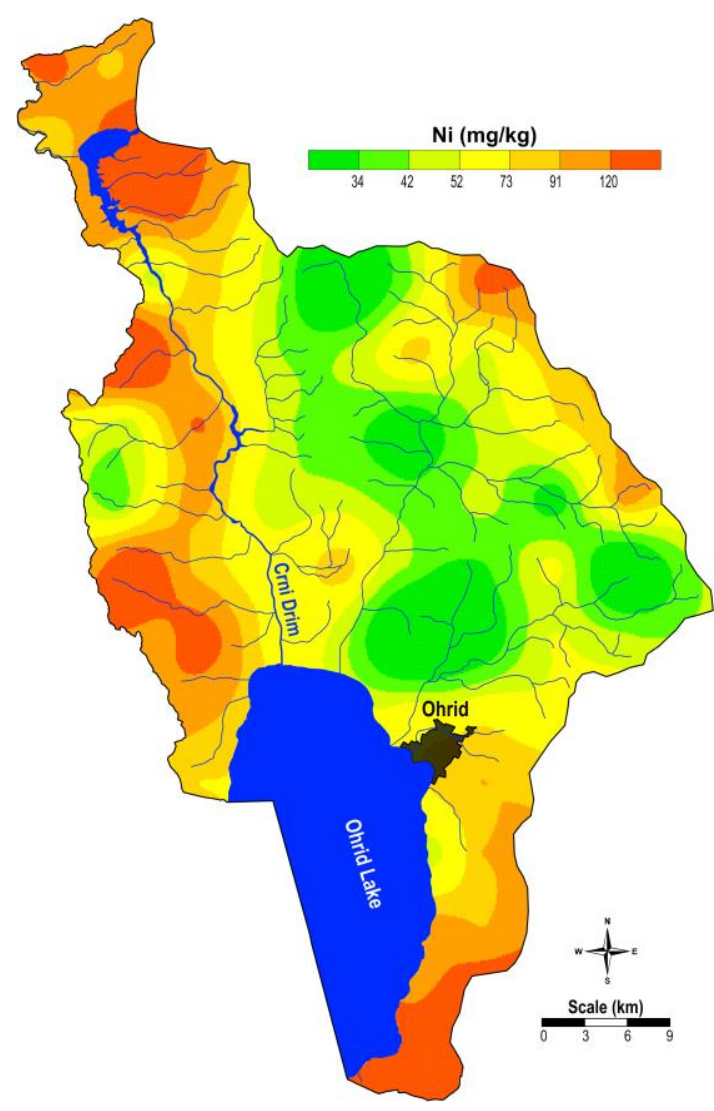

Figure 6. Spatial distribution of nickel in the Crn Drim River Basin

surface layer at the Europe $(13 \mathrm{mg} / \mathrm{kg}$ ) and Macedonia $(16 \mathrm{mg} / \mathrm{kg})$ levels. The same applies to the median in the surface layer with a value of $34 \mathrm{mg} / \mathrm{kg}$. The minimum and maximum values ranges were 8 $125 \mathrm{mg} / \mathrm{kg}$ and $11-75 \mathrm{mg} / \mathrm{kg}$, respectively. The spatial distributions of the content of $\mathrm{Pb}$ and $\mathrm{Zn}$ (Figures 8 and 9) were similar to the other elements from Factor 2 (i.e. the areas occupied by the Paleozoic and Mesozoic carbonates, as well as by Paleozoic sandstones in the northern and eastern part of the basin). According to the data presented in Table 4, the median value for $\mathrm{Pb}$ was higher in relation to the median for European and Macedonian soils, both in the topsoil and in the subsoil. In the study area, it was $31 \mathrm{mg} / \mathrm{kg}$ (range: $14-62 \mathrm{mg} / \mathrm{kg}$ ) and 29 $\mathrm{mg} / \mathrm{kg}$ (range: $5-50 \mathrm{mg} / \mathrm{kg}$ ), whereas the values for European and for Macedonian soil were 10 and 17.2 $\mathrm{mg} / \mathrm{kg}$, and 17 and $14 \mathrm{mg} / \mathrm{kg}$, respectively. The last element in this factor was zinc, whose spatial distribution is given in Figure 9. The median value for $\mathrm{Zn}$ was the same for top- and subsoil samples (130 $\mathrm{mg} / \mathrm{kg}$ ), and it was higher than in the sampled than in the European and Macedonian soil (Table 4) These findings for some parts (the town of Ohrid and its surroundings) of the investigated region could be also explained by anthropogenic influence of the urban and industrial activities [34]. 


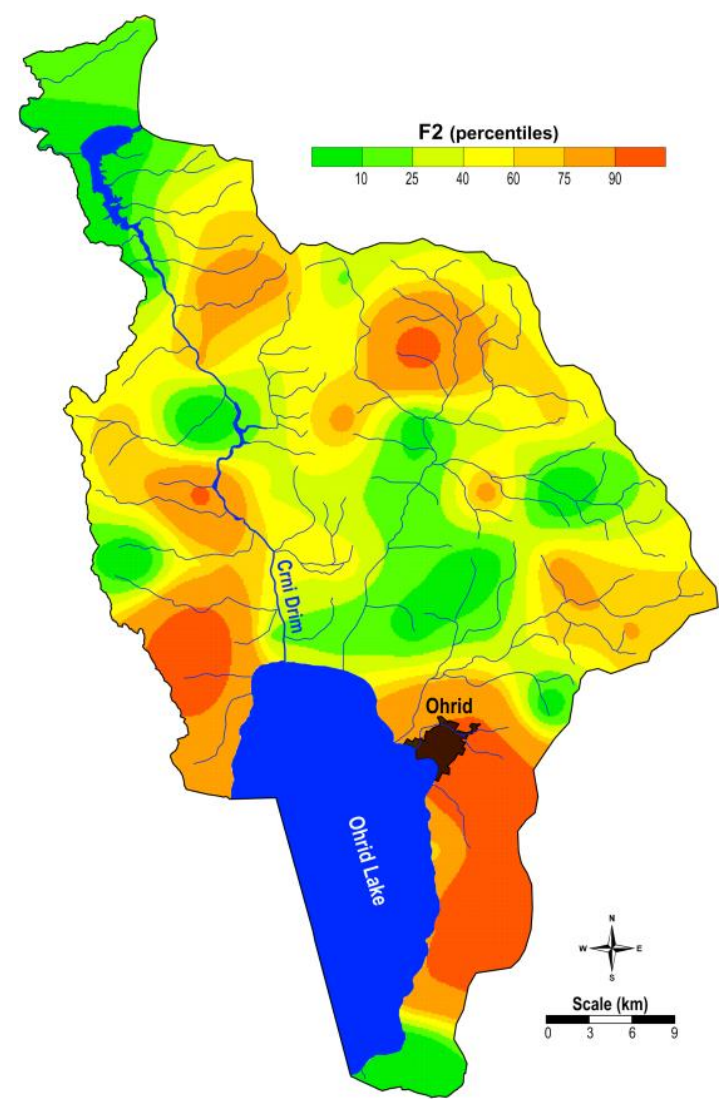

Figure 7. Spatial distribution of factor scores of $\mathrm{F} 2(\mathrm{Ag}, \mathrm{Cd}, \mathrm{Cu}, \mathrm{Pb}, \mathrm{Zn})$ in the $\mathrm{Crn}$ Drim River Basin

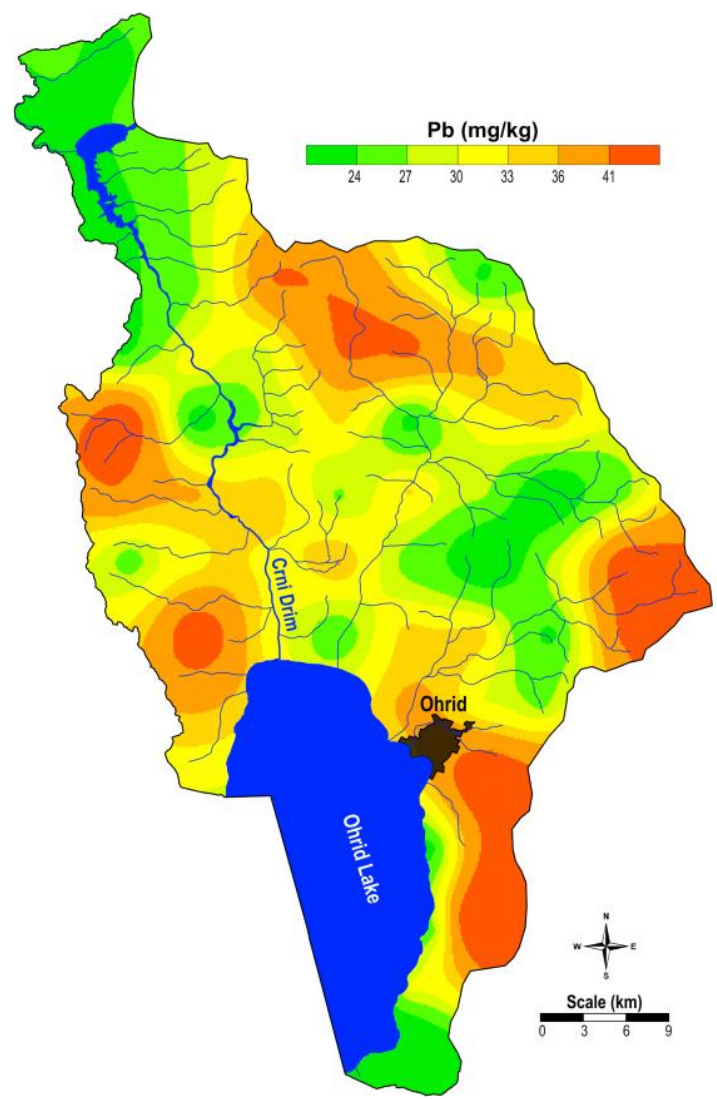

Figure 8. Spatial distribution of lead in the Crn Drim River Basin

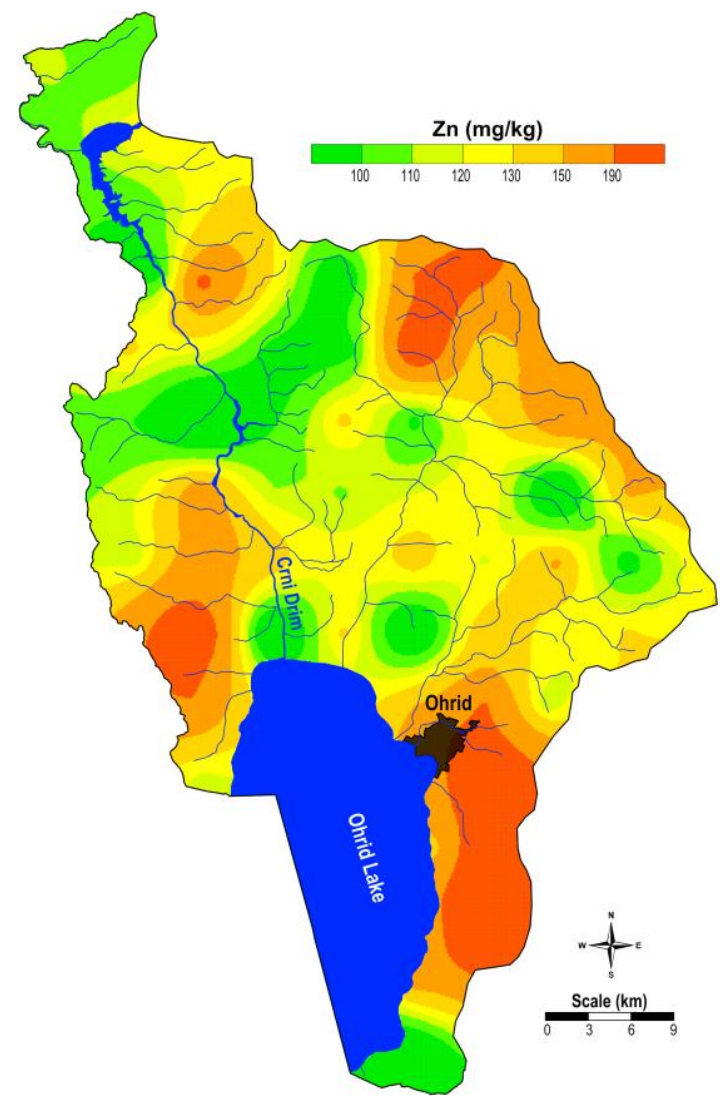

Figure 9. Spatial distribution of zinc in the Crn Drim River Basin 
Factor $3(\mathrm{Cr}, \mathrm{Fe}, \mathrm{Na}, \mathrm{Ni}, \mathrm{V})$ is a natural factor that depends on the underlying soil lithology and the spatial distribution of the factor scores. Figure 10 shows that the sources of these elements are mainly natural phenomena such as the erosion of rocks and soil chemical processes. Higher content of these elements was located in areas of Tertiary sediments in the area of Debar, while lower contents were found in the areas of Paleozoic and Mesozoic carbonates on the Galičica and Jakupica Mountains, and of Quaternary sediments in the Ohrid-Struga Valley. Typical examples of this association of elements are chromium and nickel (Figures 11 and 12). The highest values for $\mathrm{Cr}$ and $\mathrm{Ni}$ were obtained in soils around the Debar Lake and Center Župa and near the village of Slivovo). The content of $\mathrm{Cr}$ ranged from 50 to $1000 \mathrm{mg} / \mathrm{kg}$ and from 49 to 910 $\mathrm{mg} / \mathrm{kg}$, in top- and subsoil, with median values of 87 $\mathrm{mg} / \mathrm{kg}$ and $86 \mathrm{mg} / \mathrm{kg}$, respectively. Corresponding values for Ni content were: a range of 12 to 1000 $\mathrm{mg} / \mathrm{kg}$ in both layers, and medians of $61 \mathrm{mg} / \mathrm{kg}$ and $68 \mathrm{mg} / \mathrm{kg}$.

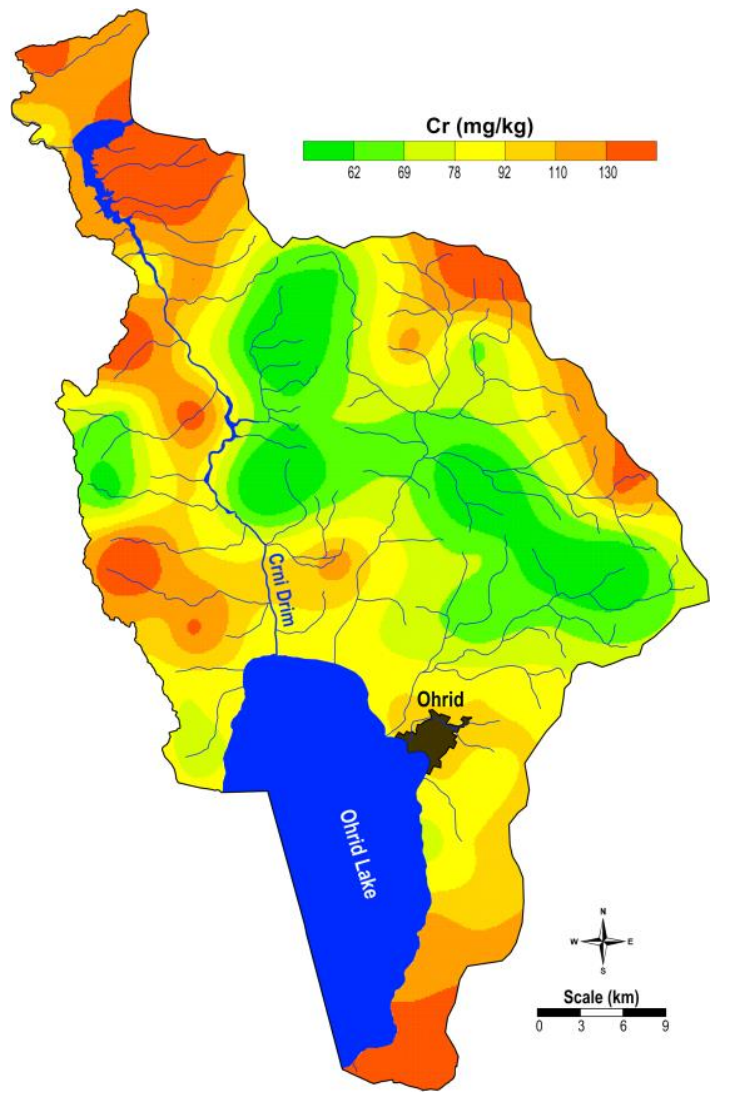

Figure 11. Spatial distribution of chromium in the Crn Drim River Basin

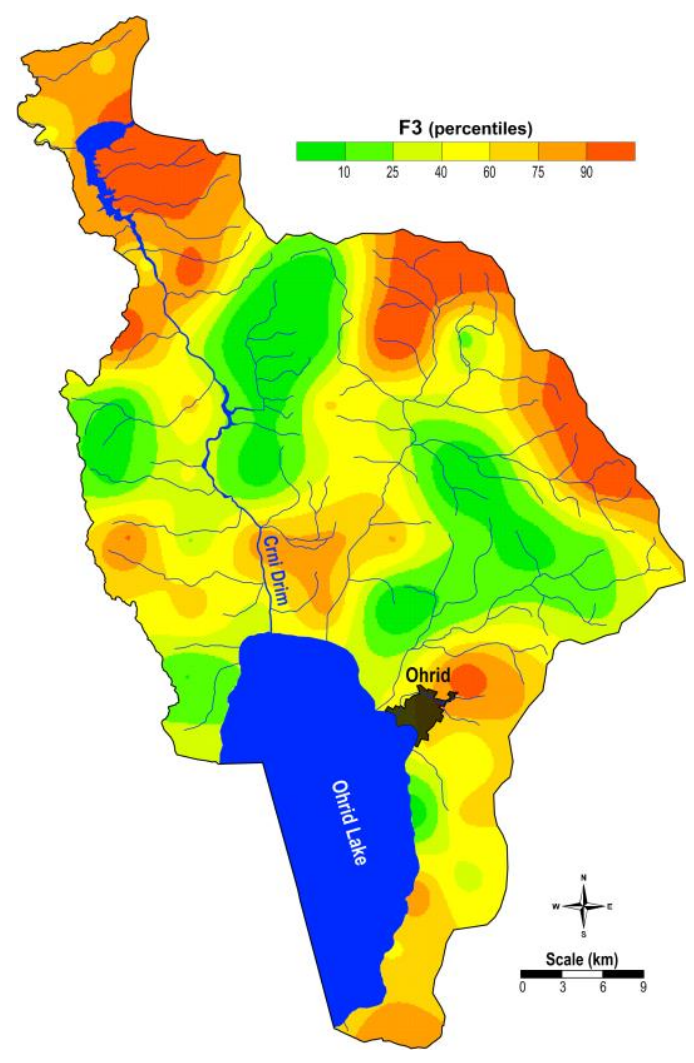

Figure 10. Spatial distribution of factor scores of $\mathrm{F} 3(\mathrm{Cr}, \mathrm{Fe}, \mathrm{Na}, \mathrm{Ni}, \mathrm{V})$ in the Crn Drim River Basin

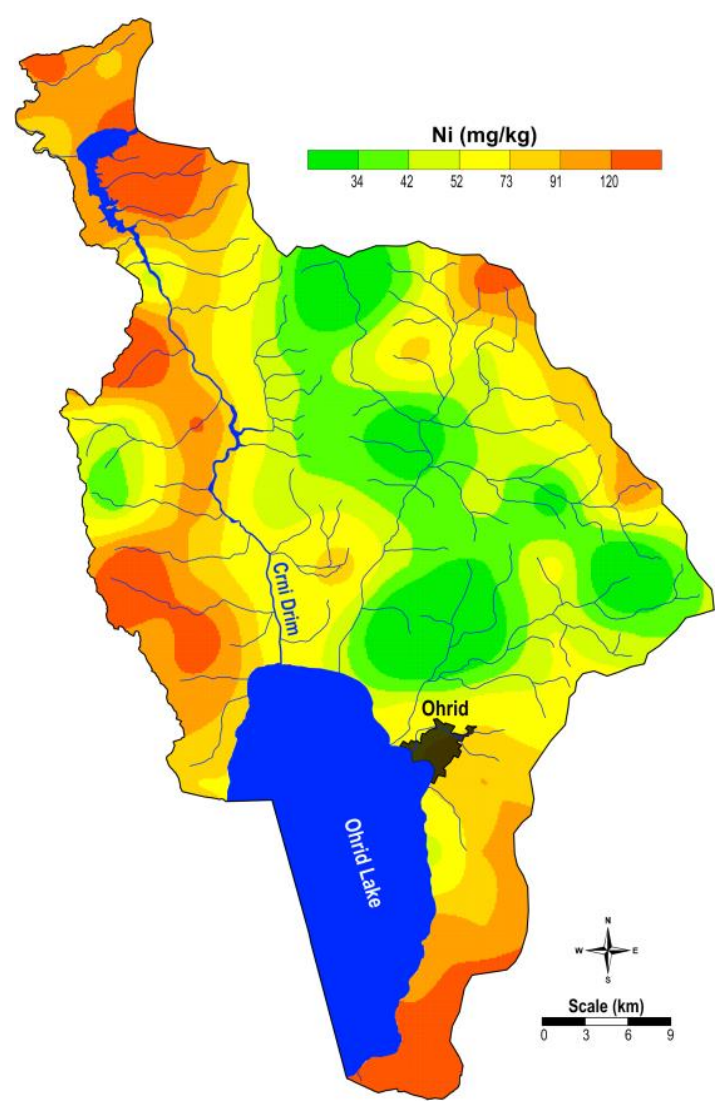

Figure 12. Spatial distribution of nickel in the Crn Drim River Basin 
Factor 4 includes $\mathrm{Al}, \mathrm{Ca}, \mathrm{Mg}$ and $\mathrm{Mn}$. The highest contents were found in the areas of Paleozoic and Mesozoic carbonates present on the Galičica and Jakupica Mountains, and on the eastern part of the basin, as well as in the areas of volcanic and magmatic rock in the western part of the area (Figure 13). This distribution was related to areas with carbonate rocks; which is very characteristic of the spatial distribution of $\mathrm{Ca}$ and $\mathrm{Mg}$ (Figures 14 and 15). The median content for $\mathrm{Mg}$ was $0.64 \%$ and $0.69 \%$ for top- and subsoil, respectively, with a range of $0.22 \%$ to $7.2 \%$ in the surface layer and $0.21 \%$ to 5.8 in the sub-layer. The median value for $\mathrm{Ca}$ was slightly smaller; $0.56 \%$ and $0.54 \%$ respectively, for the two layers. High value of $\mathrm{Ca}$ and $\mathrm{Mg}$ occured in several places: in the middle part of Galičica Mountain, but also near the village of Konjari, where the soil is predominantly over the Paleozoic and Mesozoic carbonates, near the village of Lukovo, an area dominated by magmatic rocks, southwest of the city of Struga, near the village of Radožda, located on the surface of the Paleozoic and Mesozoic carbonates, and in Upper Debarca, where Precambrian and Mesozoic schists, magmatic rocks and Paleozoic and Mesozoic carbonates are dominant.

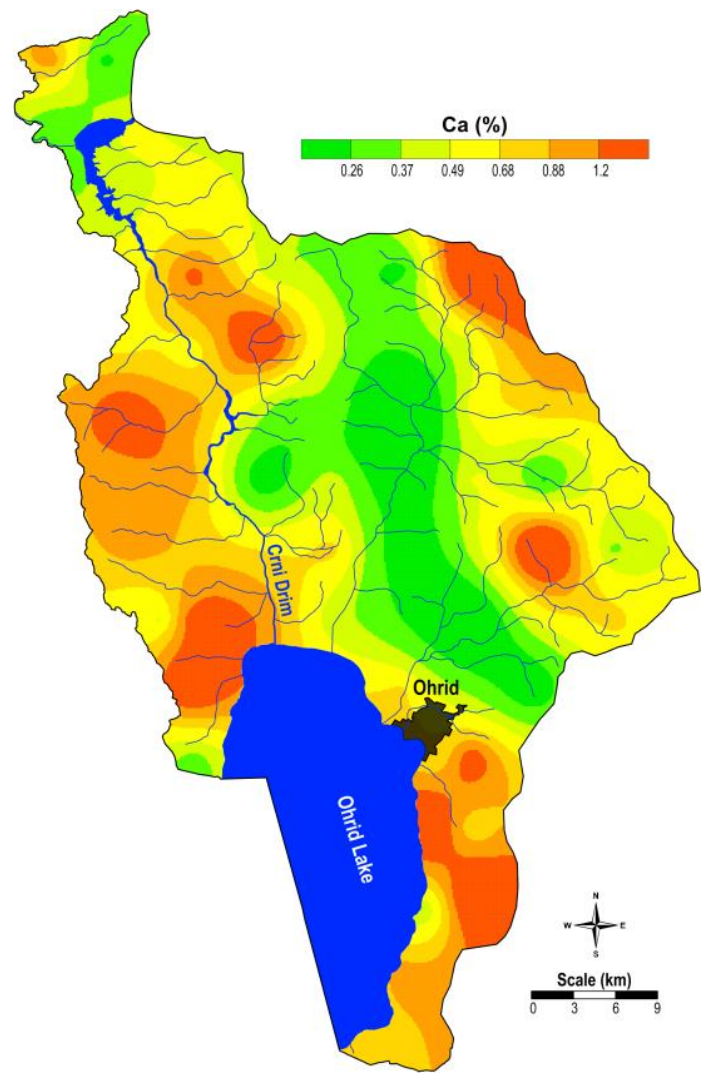

Figure 14. Spatial distribution of calcium in the Crn Drim River Basin

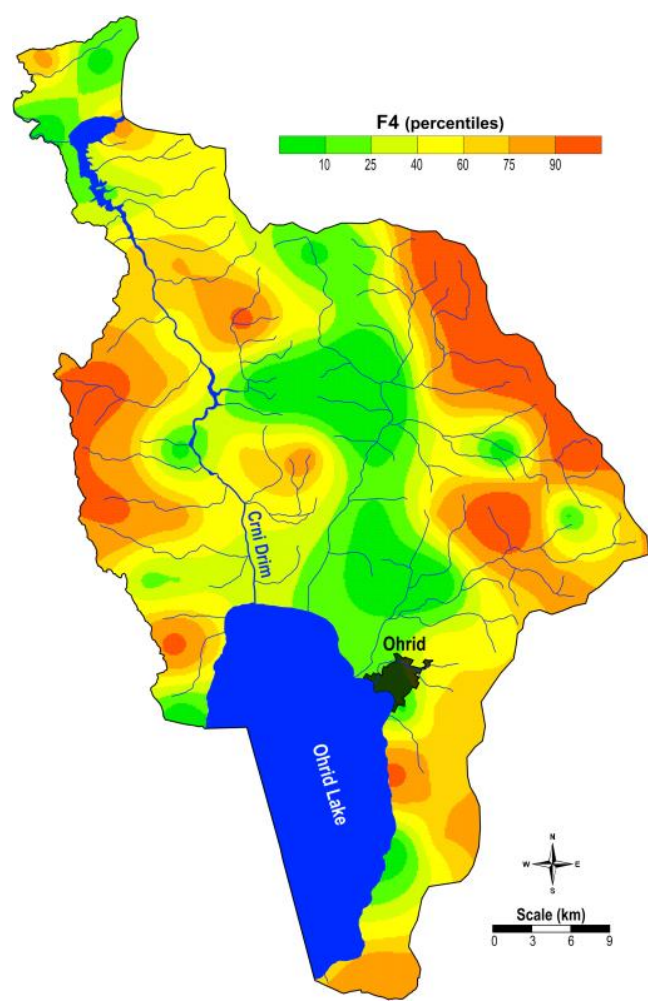

Figure 13. Spatial distribution of factor scores of F4 ( $\mathrm{Al}, \mathrm{Ca}, \mathrm{Mg}$ and $\mathrm{Mn}$ ) in the Crn Drim River Basin

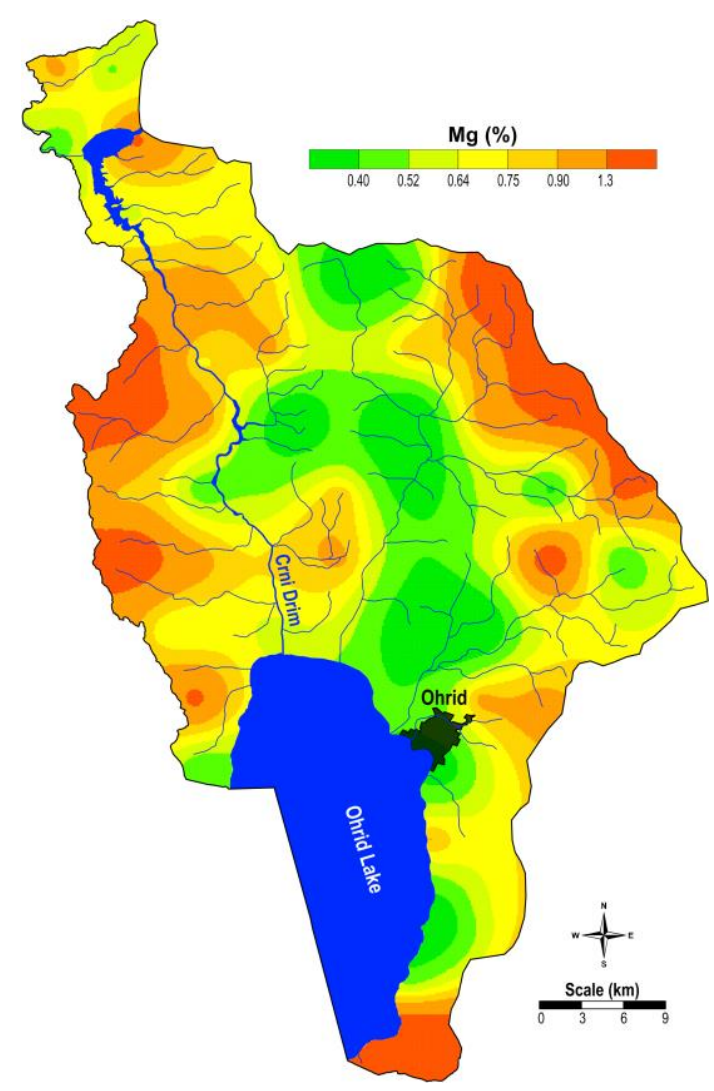

Figure 15. Spatial distribution of magnesium in the Crn Drim River Basin 
Bivariate statistics were also applied to the rare-earth elements (REE) determined by ICP-MS. The matrix of correlation coefficients is given in Table 6, where the correlation for the content of the following 14 rare elements is represented: Ce, Dy, $\mathrm{Er}, \mathrm{Eu}, \mathrm{Gd}, \mathrm{Ho}, \mathrm{La}, \mathrm{Lu}, \mathrm{Nd}, \mathrm{Pr}, \mathrm{Sm}, \mathrm{Tb}, \mathrm{Tm}$ and $\mathrm{Yb}$. It is obvious that the correlation coefficients for all of the REE were very high (from 0.75 to 1.0 ). The map of their spatial distribution is presented in Fig- ure 16. It can be noted that the highest values existed in the areas dominated by the Paleozoic and Mesozoic carbonates (in the vicinity of Velestovo and Konsko, as well as on the part of the Galičica Mountain, but also in the Struga area near the villages of Oktisi and Vevčani). There are also high values for their content in the region of the Precambrian and Paleozoic schists in Debarca, occupied by Precambrian and Paleozoic schists.

Table 6. Matrix of correlation coefficients for the rare-earth elements $(n=124)$

\begin{tabular}{|c|c|c|c|c|c|c|c|c|c|c|c|c|c|c|}
\hline Element & $\mathrm{Ce}$ & Dy & $\mathrm{Er}$ & $\mathrm{Eu}$ & $\mathrm{Gd}$ & Ho & $\mathrm{La}$ & $\mathrm{Lu}$ & $\mathrm{Nd}$ & $\operatorname{Pr}$ & $\mathrm{Sm}$ & $\mathrm{Tb}$ & $\mathrm{Tm}$ & $\mathrm{Yb}$ \\
\hline $\mathrm{Ce}$ & 1.00 & & & & & & & & & & & & & \\
\hline Dy & 0.81 & 1.00 & & & & & & & & & & & & \\
\hline $\mathrm{Er}$ & 0.75 & 0.99 & 1.00 & & & & & & & & & & & \\
\hline $\mathrm{Eu}$ & 0.90 & 0.92 & 0.88 & 1.00 & & & & & & & & & & \\
\hline Gd & 0.91 & 0.97 & 0.94 & 0.97 & 1.00 & & & & & & & & & \\
\hline Ho & 0.77 & 1.00 & 1.00 & 0.89 & 0.95 & 1.00 & & & & & & & & \\
\hline $\mathrm{La}$ & 0.93 & 0.83 & 0.79 & 0.86 & 0.90 & 0.80 & 1.00 & & & & & & & \\
\hline $\mathrm{Lu}$ & 0.81 & 0.98 & 0.98 & 0.92 & 0.96 & 0.98 & 0.82 & 1.00 & & & & & & \\
\hline $\mathrm{Nd}$ & 0.96 & 0.91 & 0.86 & 0.96 & 0.98 & 0.87 & 0.95 & 0.90 & 1.00 & & & & & \\
\hline $\operatorname{Pr}$ & 0.97 & 0.89 & 0.84 & 0.95 & 0.96 & 0.85 & 0.95 & 0.88 & 1.00 & 1.00 & & & & \\
\hline $\mathrm{Sm}$ & 0.95 & 0.94 & 0.89 & 0.97 & 0.99 & 0.90 & 0.92 & 0.93 & 0.99 & 0.99 & 1.00 & & & \\
\hline $\mathrm{Tb}$ & 0.87 & 0.99 & 0.97 & 0.95 & 0.99 & 0.98 & 0.87 & 0.97 & 0.95 & 0.93 & 0.97 & 1.00 & & \\
\hline $\mathrm{Tm}$ & 0.76 & 0.99 & 1.00 & 0.88 & 0.94 & 0.99 & 0.78 & 0.98 & 0.86 & 0.84 & 0.89 & 0.97 & 1.00 & \\
\hline $\mathrm{Yb}$ & 0.77 & 0.99 & 1.00 & 0.89 & 0.95 & 0.99 & 0.79 & 0.99 & 0.87 & 0.85 & 0.90 & 0.97 & 1.00 & 1.00 \\
\hline
\end{tabular}

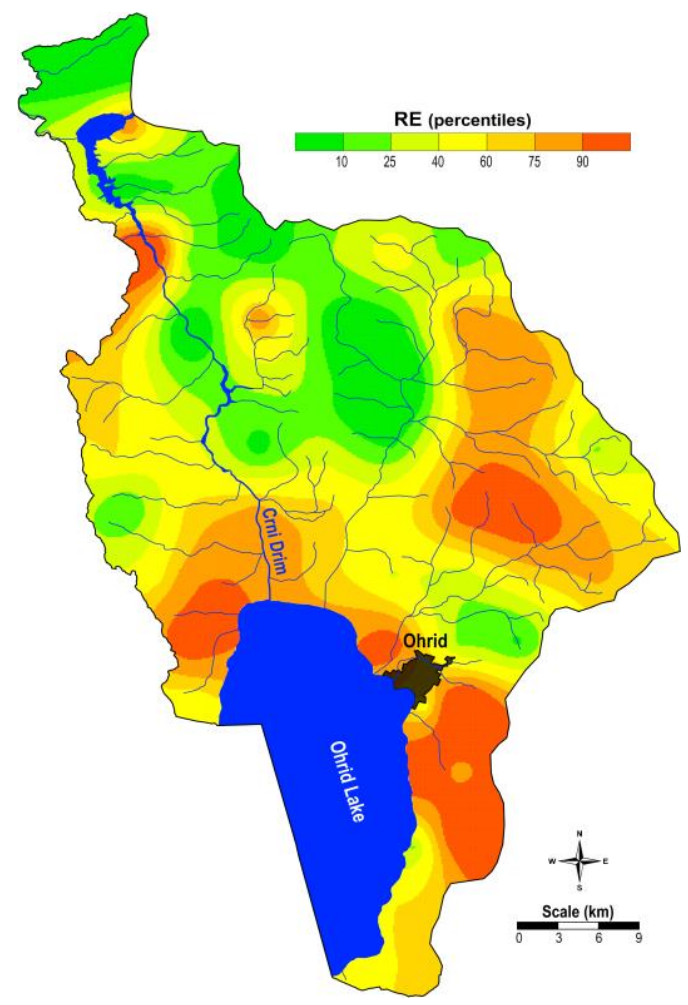

Figure 16. Spatial distribution of rare-earth elements in the Crn Drim River Basin 


\section{CONCLUSION}

The study on the distribution of sixty elements in soil from 62 locations in the Crn Drim River Basin, Republic of Macedonia, was performed. Factor analysis with the multivariate Rmethod was applied in order to show the associations between chemical elements. Four factors were obtained from applying factor analysis to the first group of the elements analysed by ICP-AES: Factor 1 (Ba and $\mathrm{K})$, Factor 2 (Ag, Cd, Cu, Ni, Pb and $\mathrm{Zn}$ ), Factor $3(\mathrm{Cr}, \mathrm{Fe}, \mathrm{Na}, \mathrm{Ni}$ and $\mathrm{V}$ ) and Factor $4(\mathrm{Al}$, $\mathrm{Ca}, \mathrm{Mg}$ and $\mathrm{Mn}$ ). Data obtained from the distribution maps of the factors and individual elements, as well as data analysis on soil samples, indicate the natural occurrence of the analysed elements as well as low concentrations of heavy metals in the studied area.

\section{REFERENCES}

[1] A. Kabata-Pendias, H. Pendias, Trace Elements in Soil and Plants, Third ed., CRC Press, Boca Raton, 2001.

[2] C. Qin, C. Luo, Y. Chen, Z. Shen, Spatial-based assessment of metal contamination in agricultural soils near an abandoned copper mine of Eastern China, Bull. Environ. Contam. Toxicol., 89 (2012), pp. 113-118.

[3] J. O. Duruibe, M. O. C. Ogwuegbu, J. N. Egwurugwu, Heavy metal pollution and human biotoxic effects, Int. J. Phys. Sci., 2 (2007), pp. 112-118.

[4] M. Hutton, C. Symon, The quantities of cadmium, lead, mercury and arsenic entering the U.K. environment from human activities, Sci. Total Environ., 57 (1986), pp. 129-150.

[5] J. O. Nriagu, A global assessment of natural sources of atmospheric trace metals, Nature, 338 (1989), pp. 47-49.

[6] D. Peplow, Environmental Impacts of Mining in Eastern Washington, Center for Water and Watershed Studies, University of Washington, Seattle, 1999.

[7] T. Mitkova, J. Mitrikeski, Soils of the Republic of Macedonia: Present Situation and Future Prospects, In: Soil Resources of Europe: second edition. R.J.A. Jones, B. Houskova, L. Montanarella \& P. Bullock, (eds). EUR $20559 \mathrm{EN}, 420$ pp, Office for Official Publications of the European Communities, Luxembourg, European Soil Bureau, Research Report No. 9, pp. 225-234, 2005.

[8] G. Filipovski, The soils of the Republic of Macedonia on Topographic Base 1: 200,000 (East of Greenwich), The Interpreter, J. Mitrikeski (ed.), Ss. Cyril and Methodius University, Institute of Agriculture, Skopje, 2015. (In Macedonian).
[9] G. Filipovski, M. Andreevski, K. Vasilevski, I. Milevski, M. Markoski, T. Mitkova J. Mitrikeski, D. Mukaetov, D. Petkovski, Pedological (Soil) Map, Ss. Cyril and Methodius University, Institute of Agriculture, Skopje, 2015. (In Macedonian).

[10] G. Filipovski, Degradation of soil as a component of the environment in the Republic of Macedonia, Macedonian Academy of Sciences and Arts, Skopje, 2003 (In Macedonian)

[11] L. Barandovski, M. Cekova, M. V. Frontasyeva, S. S. Pavlov, T. Stafilov, E. Steinnes, V. Urumov, Atmospheric deposition of trace element pollutants in Macedonia studied by the moss biomonitoring technique. Environ. Monit. Assess., 138 (2008), pp. 107-118.

[12] T. Stafilov, R. Šajn, Z. Pančevski, B. Boev, M. V. Frontasyeva, L. P. Strelkova, Heavy metal contamination of surface soils around a lead and zinc smelter in the Republic of Macedonia, $J$. Hazard. Mat., 175 (2010), pp. 896-914.

[13] T. Stafilov, R. Šajn, B. Boev, J. Cvetković, D. Mukaetov, M. Andreevski, S. Lepitkova, Distribution of some elements in surface soil over the Kavadarci region, Republic of Macedonia, Environ. Earth Sci., 61 (2010), pp. 1515-1530.

[14] B. Balabanova, T. Stafilov, K. Bačeva, R. Šajn, Biomonitoring of atmospheric pollution with heavy metals in the copper mine vicinity located near Radovıš, Republic of Macedonia. J. Environ. Sci. Health A, 45 (2010), pp. 1504-1518.

[15] L. Barandovski, M. V. Frontasyeva, T., Stafilov, R. Šajn, S. Pavlov, V. Enimiteva, Trends of atmospheric deposition of trace elements in Macedonia studied by the moss biomonitoring technique. J. Environ. Sci. Health A, 47 (2012), pp. 2000-2015.

[16] Bačeva, K., Stafilov, T., Šajn, R. \& Tănăselia, C., (2012). Moss biomonitoring of air pollution with heavy metals in the vicinity of a ferronickel smelter plant. J. Environ. Sci. Health A, 47 (2012), pp. 645-656.

[17] L. Barandovski, T. Stafilov, R. Šajn, M. V. Frontasyeva, K. Bačeva, Air pollution study in Macedonia using a moss biomonitoring technique, ICP-AES and AAS. Maced. J. Chem. Chem. Eng., 32 (2013), pp. 89-107.

[18] T. Stafilov, Environmental pollution with heavy metals in the Republic of Macedonia, Contrib. Sect. Nat. Math. Biotech. Sci. MASA, 35 (2014), pp. 81-119.

[19] L. Barandovski, M. V. Frontasyeva, T. Stafilov, R. Šajn, T. Ostrovnaya, Atmospheric deposition of trace elements in Macedonia studied by the moss biomonitoring technique, Environ. Sci. Pollut. Res., 22 (2015), pp. 16077-16097.

[20] T. Stafilov, R. Šajn, Geochemical Atlas of the Republic of Macedonia, Faculty of Natural Sciences 
and Mathematics, Ss. Cyril and Methodius University, Skopje, 2016.

[21] T. Stafilov, R. Šajn, L. Barandovski, K. Bačeva Andonovska, S. Malinovska, Moss biomonitoring air quality study of minor and trace elements in Macedonia, Air Qual. Atmos. Health, 11 (2018), pp. 137-152.

[22] B. Markoski, Cartographic definition and differentiation of the spatial parts of the valleys in the Republic of Macedonia, Bull. Dept. Phys. Geog., 2 (2005), pp. 47-66. (In Macedonian).

[23] Fishing Base for Fishing Water of the Crn Drim River Basin for the Period 2011-2016, Ministry of Agriculture, Forestry and Water Economy, Skopje, Off. Gaz. R. Macedonia, No. 66, 12 May 2011 (In Macedonian).

[24] N. Marinovski, S. Pocev, T. Dojčinoski, R. Gogoski, Ž. Andreski, P. Aslimoski, K. Angelovska, Najdoska, M. Lozanoska, J. Gavriloski, K. Marinoski, M. Ristevski, S. Saveski, Program for Plan and Development of the Southwestern Planning Region, Center for Development of Southwestern Planning Region, Struga, 2010, pp. 1-153 (In Macedonian).

[25] Pendžerkovski, J., Hadži-Mitrova, S. (1977). Interpreter of the Geological Map of SR Macedonia 1:200,000, Professional fund of the Geological Survey of Macedonia, Skopje.

[26] M. Arsovski, Tectonics of Macedonia, Faculty of Mining and Geology, Štip, pp. 1-306 (In Macedonian).

[27] N. Dumurdžanov, T. Serafimovski, B. C. Burchfiel, Evolution of the Neogene-Pleistocene Basins of Macedonia. Geological Society of America Digital Map and Chart Series 1 (accompanying notes), Boulder, Colorado, 2004, pp. 1-20.
[28] N. Dumurdžanov, T. Serafimovski, B. C. Burchfiel, Cenozoic tectonics of Macedonia and its relation to the South Balkan extensional regime, Geosphere, 1 (2005), pp. 1-22.

[29] N. Dumurdžanov, T. Ivanovski, Guidebook for the Basic Geological map of SFRJ, 1:100000, Interpreter of sheet Ohrid and Podgradec, Belgrade, 1978 (In Macedonian).

[30] R. Salminen, M. J. Batista, M. Bidovec, A. Demetriades, B. De Vivo, W. De Vos, M. Duris, A. Gilucis, V. Gregorauskiene, J. Halamic, P. Heitzmann, G. Jordan, G. Klaver, P. Klein, J. Lis, J. Locutura, K. Marsina, A. Mazreku, P. J. O'Connor, S. Å. Olsson, R. T. Ottesen, V. Petersell, J. A. Plant, S. Reeder, I. Salpeteur, H. Sandström, U. Siewers, A. Steenfelt, T. Tarvainen, Geochemical Atlas of Europe, Part 1, Background Information, Methodology and Maps, Geological Survey of Finland, Espoo, 2005.

[31] R. Šajn, Using attic dust and soil for the separation of anthropogenic and geogenic elemental distributions in an old metallurgic area (Celje, Slovenia), Geochem. Explor. Environ. Anal., 5 (2005), pp. 59-67.

[32] C. Reimann, P. Filzmoser, R. G. Garrett, Factor analysis applied to regional geochemical data: problems and possibilities, Appl. Geochem., 17 (2002), pp. 185-206.

[33] W. H. Shetaya, S. D. Young, M.J. Watts, E. L. Ander, E. H. Bailey, Iodine dynamics in soils, $\mathrm{Ge}$ ochim. Cosmochim. Acta, 77 (2012), pp. 457-473.

[34] S. Vasilevska, T. Stafilov, R. Šajn, Distribution of chemical elements in surface water from Crn Drim River Basin, Republic of Macedonia, Water Res. Manag., 8 (2018), pp. 3-15.

\title{
ДИСТРИБУЦИЈА НА ХЕМИСКИ ЕЛЕМЕНТИ ВО ПОЧВИТЕ ОД СЛИВОТ НА РЕКАТА ЦРН ДРИМ, РЕПУБЛИКА МАКЕДОНИЈА
}

\author{
Трајче Стафилов $^{1 *}$, Роберт Шајн ${ }^{2}$, Ивана Мицковска ${ }^{1}$
}

\author{
${ }^{1}$ Институт за хемија, Природно-математички факултет, Универзитет „Св. Кирил и Методиј“, \\ Скопје, Република Македонија \\ ${ }^{2}$ Геолошки завод на Словенија, Љубљана, Словенија
}

Целта на истражувањето е утврдување на дистрибуцијата на хемиските елементи во површинските и потповршинските почви од регионот на сливот на реката Црн Дрим, Република Македонија, со посебно внимание на природното и антропогеното потекло на елементите. За таа цел, со мрежа за земање на примероци од $5 \times 5 \mathrm{~km}$, земени се вкупно 124 примероци почва од 62 локации (површински и потповршински). Анализирани се вкупно 60 елементи, од кои 18 елементи (Ag, Al, B, Ba, Ca, Cr, Cu, Fe, K, Li, Mg, Mn, Na, Ni, P, $\mathrm{Pb}, \mathrm{V}$ и $\mathrm{Zn}$ ) се анализирани со примена на атомската емисиона спектрометрија со индуктивно спрегната плазма (ICP-AES) а останатите 42 елементи со примена на масената спектрометрија со индуктивно спрегната плазма (ICP-MS). Добиените резултати се обработени со мултивариатната статистичка метода. Факторната анализа на податоците добиени со ICP-AES дава четити геогени фактори: F1 (Ba и K); F2 (Ag, Cd, Cu, Ni, Pb и Zn), F3 (Cr, 
$\mathrm{Fe}, \mathrm{Na}, \mathrm{Ni}$ и V) и F4 (Al, Ca, Mg и Mn). Податоците од анализата на примероците и од картите на дистрибуција покажуваат поврзаност со литогенезата на испитуваните елементи и со релативно ниски содржини на тешките метали во испитуваното подрачје.

Клучни зборови: почви; слив на реката Црн Дрим; Република Македонија; мултиваријатна статистика; геохемиско мапирање 